\title{
Packet-Based Modeling of Reed-Solomon Block-Coded Correlated Fading Channels Via a Markov Finite Queue Model
}

\author{
Cecilio Pimentel and Fady Alajaji, Senior Member, IEEE
}

\begin{abstract}
We consider the transmission of a Reed-Solomon (RS) code over a binary modulated time-correlated flat Rician fading channel with hard-decision demodulation. We define a binary packet (symbol) error sequence that indicates whether an RS symbol is successfully transmitted across the discrete (fading) channel whose input enters the modulator and whose output exits the demodulator. We then approximate the packet error sequence of the discrete channel (DC) using the recently developed queue-based channel (QBC), which is a simple finite-state Markov channel model with $M$ th-order Markovian additive noise. In other words, we use the $\mathrm{QBC}$ to model the binary DC at the packet level. We propose a general framework for determining the probability of codeword error (PCE) for QBC models. We evaluate the modeling accuracy by comparing the simulated PCE for the DC with the numerically evaluated PCE for the QBC. Modeling results identify accurate low-order QBC models for a wide range of fading conditions and reveal that modeling the DC at the packet level is an efficient tool for nonbinary coding performance evaluation over binary channels with memory.
\end{abstract}

Index Terms-Burst-noise channels, finite-state Markov channels (FSMCs), packet-error sequence, probability of codeword error (PCE), queue-based channel (QBC), Reed-Solomon (RS) codes, Rician fading.

\section{INTRODUCTION}

$\mathbf{T}$ HE DEVELOPMENT of binary additive (first-order) Markov noise channel (BAMNC) models to represent the successes and failures of the transmission of information packets over correlated flat-fading channels was considered in [1]-[4]. In these works, the packet-error (i.e., noise) process is constructed by taking into consideration the packet length, the parameters of the fading channel, the modulation/demodulation/diversity techniques, and the coding/ decoding schemes (if coded transmission is considered). It is

Manuscript received May 20, 2008; revised November 14, 2008. First published February 10, 2009; current version published August 14, 2009. This work was supported in part by the Natural Sciences and Engineering Research Council of Canada and in part by the National Council for Research and Development $(\mathrm{CNPq})$ of Brazil. This paper was presented in part at the IEEE Workshop on Signal Processing Advances in Wireless Communications (SPAWC'2008), Recife, Brazil, July 2008. The review of this paper was coordinated by Dr. A. Ghrayeb.

C. Pimentel is with the Department of Electronics and Systems, Federal University of Pernambuco, Recife, PE 50711-970, Brazil (e-mail: cecilio@ufpe.br).

F. Alajaji is with the Department of Mathematics and Statistics, Queen's University, Kingston, ON K7L 3N6, Canada (e-mail: fady@mast.queensu.ca).

Digital Object Identifier 10.1109/TVT.2009.2014957 assumed in [1]-[4] that the fading process is constant within the packet duration, which makes these methodologies suitable for slowly fading channels. Binary finite-state Markov channel (FSMC) models with more than two states were also proposed to model the information blocks produced by the physical layer of a coded communication system [5], [6]. Such FSMC models characterize the residual correlation structure of the successes and failures of consecutive blocks of information symbols at the output of the decoder. In this scenario, closed-form expressions for the performance analysis of error-correcting codes on FSMCs [7]-[9] may be employed for further protocol performance evaluation in a higher layer network.

Binary FSMC models were also used to characterize the bit error process of the discrete communication fading channel (from the input of the modulator to the output of the demodulator) [10]-[16]. In this case, the FSMC model allows analytical coding performance evaluation with arbitrary fading rates and, hence, provides the tools for the construction of powerful coding techniques that effectively exploit the channel statistical memory, as opposed to ignoring it via interleaving. Indeed, codes that were designed to take into account the channel memory can considerably outperform traditional codes designed for the equivalent memoryless channel (realized via perfect interleaving) [17]-[19]. This is information theoretically justified by the fact that, for a wide class of information stable channels (e.g., channels with additive stationary ergodic noise), memory increases capacity (e.g., see [20]).

In this paper, we consider the transmission of Reed-Solomon (RS) codes over hard-decision binary frequency-shift keying (BFSK) demodulated time-correlated flat Rayleigh and Rician fading channels. RS codes are nonbinary error-correcting block codes of considerable importance in transmission systems operating over fading channels [21]-[26]. Due to their symbol [an element of the Galois field $\mathrm{GF}\left(2^{b}\right)$ ] orientation, RS codes are well suited to an environment where errors occur in bursts. The binary communication channel from the input of the modulator to the output of the demodulator is herein referred to as the discrete channel (DC) model. We construct a binary packet error process for the DC model from its bit error process, where the packet (or symbol) length is equal to $b$ (which is the length of the binary representation of the RS field element). Hereinafter, we refer to a packet as a binary sequence of length $b$. In this case, the $k$ th packet error bit is equal to 0 whenever the $k$ th RS symbol is successfully transmitted across the DC model. 
Otherwise, the packet error bit is equal to 1 . The objectives of this work are twofold.

1) Develop accurate FSMC models for the DC (symbol) packet-error process. This is an extension of prior works where the DC is modeled via FSMCs at the bit error process level [10]-[16].

2) Provide the tools for evaluating the probability of codeword error (PCE) for nonbinary block codes over our FSMC models under bounded distance decoding.

We will show that the derivation of the recently introduced queue-based channel (QBC) model [20] at the packet level facilitates both the DC modeling and its RS coding performance analysis.

To achieve the first objective, we approximate the binary packet-error process of the DC model using the QBC model, which is a simple FSMC model. Specifically, the QBC is a stationary $M$ th-order additive Markov noise channel with only four parameters [20]. Closed-form expressions for several statistics of the QBC model with arbitrary memory order $M$ (e.g., capacity, autocorrelation function (ACF), and block error probability) are provided in [20]. It should be noted that the QBC has several interesting attributes. These include its mathematical tractability for the design and analysis of coding systems over channels with memory (due to the closedform expressions for its statistical and information-theoretic quantities) while being an accurate model (at the bit level) for the Gilbert-Elliott channel [20] and the class of time-correlated Rician fading channels [11]. Note also that the BAMNC with a nonnegative noise correlation coefficient is a special case of the QBC. (It is obtained by setting the memory order to $M=1$.) The QBC at the packet level (i.e., at the symbol level) generates a binary sequence that indicates whether an RS symbol is successfully transmitted across the DC model and captures the correlation among consecutive RS symbols within a codeword. Under this setup, we investigate the appropriateness of the QBC in modeling the DC packet-error process.

Our contributions in the modeling part of this paper are given as follows: We first identify the range of fading parameters for which the BAMNC at the packet level is accurate. For the fading regime where the BAMNC is not accurate (slow fading), we generalize the results obtained in [4] by developing an expression for the probability of a binary packet-error sequence of length $n$ of the DC model with Rician fading under the assumption of constant (quasi-static) fading within a packet to parametrize higher order Markovian QBC models in slow-fading environments. Modeling results identify the QBC parameters for several fading conditions and reveal that low-order QBCs $(M \leq 4)$ provide a good fit for the packet-error process of the DC model, as opposed to higher order QBC models that are required to model the DC error process at the bit level for slow fading [11].

The accuracy of the QBC approximation is measured in terms of ACF and PCE. Under bounded distance decoding, the PCE for RS codes is obtained from the probability of $m$ symbol (packets) errors in a block with a length of $n$ symbols, which is denoted by $P_{s}(m, n)$. Methods for calculating $P_{s}(m, n)$ for the binary transmission of nonbinary block codes with symbols over $\operatorname{GF}\left(2^{b}\right)$ are proposed in [26] and [27]. However, the PCE performance analysis of binary block codes over FSMCs at the bit level is simpler and requires the calculation of the probability that the FSMC generates $m$ erroneous bits in a block with a length of $n$ bits, i.e., $P(m, n)$. It is worth mentioning that the methods developed in the literature for calculating $P(m, n)$ for some classes of FSMC models such as the BAMNC [9], [28], the Gilbert-Elliott channel [12], [29], and the simplified Fritchman channel [30] can readily be applied to study the PCE performance of RS codes over FSMCs at the packet level. In the latter case, the code's field size is a parameter of the model and is not considered in the $P(m, n)$ calculation. Thus, the development of FSMC models at the packet level, such as the QBC modeling studied in this paper, can significantly simplify the performance analysis of RS codes over channels with memory. Packet error modeling and RS coding performance evaluation in magnetic storage channels were also recently considered in [31].

Our contributions toward the second objective are given as follows: a novel closed-form formula for $P(m, n)$ that can be used with the BAMNC, which is much simpler than that derived in [9, eq. (39)]. In addition, an iterative matrix-based algorithm for computing $P(m, n)$ for general binary FSMC models is proposed and applied for QBC models with $M>1$. The proposed algorithm is easy to implement using a symbolic manipulation program and takes advantage of the sparse structure of the transition probability matrix of the QBC to reduce the computational complexity.

The rest of this paper is organized into six sections. Section II describes the communication system and the generation of the packet error process. Preliminaries on the QBC models are also provided in this section. The derivation of packet error statistics required for channel modeling is presented in Section III. The QBC parameter estimation for best fitting the DC at the packet level is discussed in Section IV. Section V presents a matrix-based methodology for computing $P(m, n)$ for general FSMC models. In particular, a new closed-form formula for the BAMNC is derived. Numerical fitting results in terms of PCE are provided in Section VI. Conclusions are given in Section VII.

\section{COMmunicATION SySTEM}

We consider an $(n, k)$ RS-coded communication system with RS symbols over Galois field $\mathrm{GF}\left(2^{b}\right)$, whose codewords are of length $n=2^{b}-1$ symbols and contain $k$ information symbols. The code can correct up to $t=\lfloor(n-k) / 2\rfloor$ symbols (under bounded distance decoding), where $\lfloor x\rfloor$ is the greatest integer that is less than or equal to $x$. Each symbol in $\operatorname{GF}\left(2^{b}\right)$ is mapped to a binary $b$-tuple (the vector space representation of the corresponding field element) and transmitted across a binary (binary-input, binary-output) DC.

The DC model, as shown in Fig. 1, is composed of a BFSK modulator, a time-correlated flat Rician fading channel with additive white Gaussian noise, and a hard quantized demodulator. The complex envelope of the fading process $\tilde{G}(t)$ is a complex wide-sense stationary Gaussian process with normalized second moment, i.e., $\mathbf{E}\left[|\tilde{G}(t)|^{2}\right]=1$ (where $\mathbf{E}[\cdot]$ denotes 


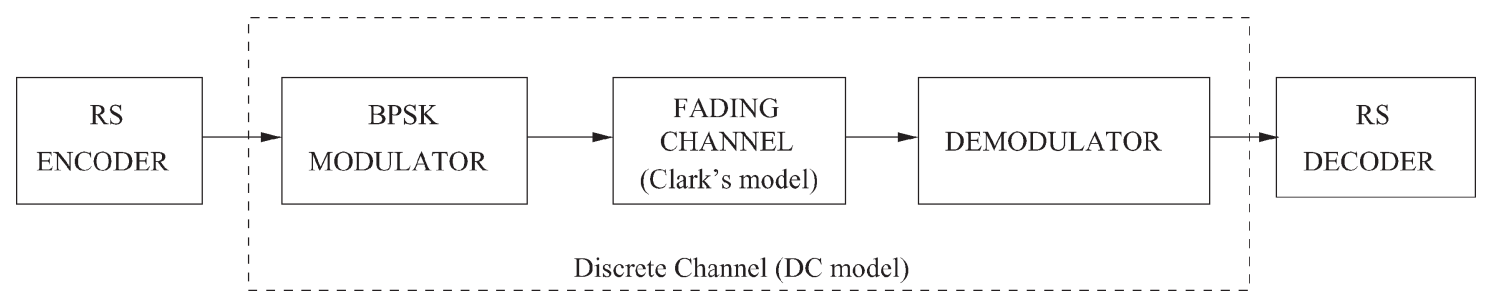

Fig. 1. Communication system with a binary DC.

the expectation), and covariance function given by Clarke's model

$$
C(\tau)=\left(\frac{1}{1+K_{R}}\right) J_{0}\left(2 \pi f_{D} \tau\right)
$$

where $J_{0}(x)$ is the zero-order Bessel function of the first kind, $f_{D}$ is the maximum Doppler frequency, and $K_{R}$ is the Rician factor. We define a binary error process $\left\{E_{k}\right\}_{k=1}^{\infty}$, where $E_{k}=$ 0 indicates no bit error at the $k$ th signaling interval of length $T$ and $E_{k}=1$ indicates a bit error. The probability of an error sequence of length $n, e^{n}=e_{1}, e_{2}, \ldots, e_{n}$ at the bit level for the DC model is denoted by $P_{\mathrm{DC}}\left(e^{n}\right)$ and is given by [32]

$$
\begin{aligned}
P_{\mathrm{DC}}\left(e^{n}\right)= & \sum_{l_{1}=e_{1}}^{1} \cdots \sum_{l_{n}=e_{n}}^{1}\left(\prod_{k=1}^{n} \frac{(-1)^{l_{k}+e_{k}}}{l_{k}+1}\right) \mathbf{E}\left[e^{-\frac{E_{s}}{N_{0}} \mathbf{G}^{\dagger} \mathbf{F G}}\right] \\
= & \sum_{l_{1}=e_{1}}^{1} \cdots \sum_{l_{n}=e_{n}}^{1}\left(\prod_{k=1}^{n} \frac{(-1)^{l_{k}+e_{k}}}{l_{k}+1}\right) \\
& \times \frac{\exp \left\{-\frac{E_{s}}{N_{0}} K_{R} \mathbf{1}^{T} \mathbf{F}\left(\left(K_{R}+1\right) \mathbf{I}+\frac{E_{s}}{N_{0}} \overline{\mathbf{C F}}\right)^{-1} \mathbf{1}\right\}}{\operatorname{det}\left(\mathbf{I}+\frac{E_{s}}{N_{0}}\left(1+K_{R}\right)^{-1} \overline{\mathbf{C}} \mathbf{F}\right)}
\end{aligned}
$$

where $\mathbf{G}$ is a column vector composed of $n$ complex Gaussian random variables $\tilde{G}_{i}, i=1, \ldots, n ; E_{s} / N_{0}$ is the signal-tonoise ratio; the $(i, j)$ th entry of the normalized $n \times n$ covariance matrix $\overline{\mathbf{C}}$ is $J_{0}\left(2 \pi f_{D}|i-j| T\right), 1 \leq i, j \leq n$; $\mathbf{F}$ is a diagonal matrix defined as $\mathbf{F}=\operatorname{diag}\left(l_{1} /\left(l_{1}+1\right)\right), \ldots,\left(l_{n} /\right.$ $\left.\left(l_{n}+1\right)\right) ; \mathbf{1}$ is a length- $n$ column vector of ones; $\mathbf{I}$ is the $n \times n$ identity matrix; and superscripts $[\cdot]^{T}$ and $[\cdot]^{\dagger}$ indicate the transpose and transpose conjugate of a matrix, respectively.

To construct the binary success/failure process $\left\{\beta_{k}\right\}_{k=1}^{\infty}$ of the transmitted packets (sequences of $b$ bits) of the DC model, the binary error process $\left\{E_{k}\right\}_{k=1}^{\infty}$ is divided into packets of length $b$. Event $\beta_{k}=0$ indicates the successful transmission of the $k$ th packet, i.e., sequence $E_{(k-1) b+1}, \ldots, E_{k b}$ is an allzero sequence (which is denoted by $0^{b}$ ), and $\beta_{k}=1$ indicates a packet error. (At least one bit in this packet is incorrectly decoded.) Thus, each binary random variable $\beta_{k}$ is a function of sequence $E_{(k-1) b+1}, \ldots, E_{k b}$, and it is binary packet-error process $\left\{\beta_{k}\right\}_{k=1}^{\infty}$ that the QBC model will herein attempt to emulate. Thus, sequence $\left\{\beta_{k}\right\}_{k=1}^{\infty}$ specifies the DC model at the packet level.

\section{A. QBC Model}

The QBC model generates a binary $M$ th-order stationary ergodic Markov noise process $\left\{Z_{k}\right\}_{k=1}^{\infty}$ using a finite queue [20]. The model is defined in terms of four parameters, i.e., the size of the queue $M$, bit error rate (BER) $p=\operatorname{Pr}\left(Z_{k}=1\right)$, and correlation parameters $\varepsilon$ and $\alpha$, where $0 \leq \varepsilon<1$ and $\alpha \geq 0$. We briefly describe the generation of the noise symbol at the $k$ th interval. More details are provided in [20]. First, one of two parcels (an urn and a queue of size $M$ ) are selected with probability distribution $\{\varepsilon, 1-\varepsilon\}$. If the urn is selected, the model generates an error $\left(Z_{k}=1\right)$ with probability $p$. If the queue is selected, a binary noise symbol is selected with a probability distribution that depends on $M$ and parameter $\alpha$. ( $\alpha$ determines the bias for operating on the last cell of the queue of length $M$ and is equal to 1 when $M=1$ [20].) The state process of the QBC $\left\{S_{k}\right\}_{k=1}^{\infty}$, where $S_{k} \triangleq\left(Z_{k}, Z_{k-1}, \ldots, Z_{k-M+1}\right)$, is a first-order Markov process with $2^{M} \times 2^{M}$ transition probability matrix $\mathbf{P}=\left[p_{i j}\right]$ given by [20, eq. (4)] and state stationary distribution column vector $\Pi=\left[\pi_{i}\right]$ given by [20, eq. (5)].

We define two $2^{M} \times 2^{M}$ matrices $\mathbf{P}(0)$ and $\mathbf{P}(1), \mathbf{P}(0)+$ $\mathbf{P}(1)=\mathbf{P}$, where the $(i, j)$ th entry of matrix $\mathbf{P}(z)$ is the probability that the model generates an error bit $z$ when the QBC state process transitions from states $i$ to $j$. For the QBC, the first $2^{M-1}$ columns of $\mathbf{P}(0)$ are exactly the same as those of $\mathbf{P}$, whereas the remaining $2^{M-1}$ columns are zeros. Similarly, the first $2^{M-1}$ columns of $\mathbf{P}(1)$ are all zeros, whereas the remaining $2^{M-1}$ columns are exactly the same as those of $\mathbf{P}$. Channel noise block probability $\operatorname{Pr}\left(Z_{1}=z_{1}, Z_{2}=z_{2}, \ldots, Z_{n}=z_{n}\right)=$ $\operatorname{Pr}\left(Z^{n}=z^{n}\right)$ is expressed in the following (see also [20]).

- For block length $n \leq M, \operatorname{Pr}\left(Z^{n}=z^{n}\right)$ is given by (3), shown at bottom of the page, where $d_{a}^{b}=z_{b}+z_{b-1}+$ $\cdots+z_{a}\left(d_{a}^{b}=0\right.$ if $\left.a>b\right)$, and $\prod_{j=0}^{a}(\cdot) \triangleq 1$ if $a<0$.

$$
P_{\mathrm{QBC}}\left(z^{n}\right) \triangleq \operatorname{Pr}\left(Z^{n}=z^{n}\right)=\frac{\prod_{j=0}^{n-d_{1}^{n}-1}\left[j \frac{\varepsilon}{M-1+\alpha}+(1-\varepsilon)(1-p)\right] \prod_{j=0}^{d_{1}^{n}-1}\left[j \frac{\varepsilon}{M-1+\alpha}+(1-\varepsilon) p\right]}{\prod_{j=M-n}^{M-1}\left[1-(\alpha+j) \frac{\varepsilon}{M-1+\alpha}\right]}
$$


- For block length $n \geq M+1$

$$
\begin{aligned}
P_{\mathrm{QBC}}\left(z^{n}\right) \triangleq & \operatorname{Pr}\left(Z^{n}=z^{n}\right) \\
= & L^{(M)} \prod_{i=M+1}^{n}\left[\left(d_{i-M+1}^{i-1}+\alpha z_{i-M}\right)\right. \\
& \left.\times \frac{\varepsilon}{M-1+\alpha}+(1-\varepsilon) p\right]^{z_{i}} \\
& \times\left\{\left[\left(M-1-d_{i-M+1}^{i-1}\right)+\alpha\left(1-z_{i-M}\right)\right]\right. \\
& \left.\quad \times \frac{\varepsilon}{M-1+\alpha}+(1-\varepsilon)(1-p)\right\}^{1-z_{i}}
\end{aligned}
$$

where $L^{(M)}$ is given by the expression shown at the bottom of the page.

The $\mathrm{ACF}$ of the $\mathrm{QBC}$, i.e., $R_{\mathrm{QBC}}[m]=\mathbf{E}\left[Z_{i} Z_{i+m}\right]$, satisfies the second expression shown at the bottom of the page [20]. The correlation coefficient of a binary stationary process is defined as

$$
\text { Cor } \triangleq \frac{R[1]-\operatorname{Pr}^{2}(1)}{\operatorname{Pr}(1)(1-\operatorname{Pr}(1))} .
$$

The correlation coefficient for the $\mathrm{QBC}, \mathrm{Cor}_{\mathrm{QBC}}$, is a nonnegative quantity given by [20]

$$
\operatorname{Cor}_{\mathrm{QBC}}=\frac{\frac{\varepsilon}{M-1+\alpha}}{1-(M-2+\alpha) \frac{\varepsilon}{M-1+\alpha}} .
$$

The BAMNC with nonnegative noise correlation coefficient is a special case of the $\mathrm{QBC}$ with $M=\alpha=1$ and noise correlation coefficient $\varepsilon$. Its transition probability matrix is written as

$$
\mathbf{P}=\left[\begin{array}{cc}
\varepsilon+(1-\varepsilon)(1-p) & (1-\varepsilon) p \\
(1-\varepsilon)(1-p) & \varepsilon+(1-\varepsilon) p
\end{array}\right]
$$

In particular, the ACF of the BAMNC is given by

$$
R_{\mathrm{BAMNC}}[m]=p^{2}+\varepsilon^{m} p(1-p), \quad \text { for } m \geq 1 .
$$

Next, we derive some statistics of the packet-error process $\left\{\beta_{k}\right\}_{k=1}^{\infty}$ of the DC model in terms of (2). These statistics will be used in Section IV to derive QBC models for the DC model at the packet level, i.e., approximate $\left\{\beta_{k}\right\}_{k=1}^{\infty}$ by $\left\{Z_{k}\right\}_{k=1}^{\infty}$.

\section{Derivation of DC Packet-Error Statistics}

\section{A. Packet-Error Correlation Coefficient}

The probability of a packet error of the DC model, which is denoted by $\mathrm{PPE}_{\beta}$, is given by

$$
\operatorname{PPE}_{\beta}=\operatorname{Pr}\left(\beta_{i}=1\right)=1-\operatorname{Pr}\left(\beta_{i}=0\right)=1-P_{\mathrm{DC}}\left(0^{b}\right)
$$

where $P_{\mathrm{DC}}\left(0^{b}\right)$ is obtained from (2). Let $\Omega^{m-1}$ denote the set of all binary sequences of length $m-1$ and $\omega$ denote a particular sequence in $\Omega^{m-1}$. The ACF of a binary stationary process may be expressed as $R[m]=\operatorname{Pr}\left(b_{k}=1, b_{k+m}=1\right)$. We then compute this probability equivalently as

$$
R[m] \triangleq \operatorname{Pr}\left(1 \Omega^{m-1} 1\right)=\sum_{\omega \in \Omega^{m-1}} P\left(b_{k}=1, \omega, b_{k+m}=1\right) .
$$

It is convenient to express $R[m]$ in terms of $\operatorname{Pr}\left(0 \Omega^{m-1} 0\right)$. First, note that

$$
\begin{aligned}
\operatorname{Pr}(0) & =\operatorname{Pr}\left(0 \Omega^{m}\right)=\operatorname{Pr}\left(0 \Omega^{m-1} 1\right)+\operatorname{Pr}\left(0 \Omega^{m-1} 0\right) \\
& =\operatorname{Pr}\left(\Omega^{m} 0\right)=\operatorname{Pr}\left(0 \Omega^{m-1} 0\right)+\operatorname{Pr}\left(1 \Omega^{m-1} 0\right) .
\end{aligned}
$$

We substitute $\operatorname{Pr}\left(0 \Omega^{m-1} 1\right)=\operatorname{Pr}\left(1 \Omega^{m-1} 0\right)=\operatorname{Pr}(0)-$ $\operatorname{Pr}\left(0 \Omega^{m-1} 0\right)$ into

$\operatorname{Pr}\left(0 \Omega^{m-1} 0\right)+\operatorname{Pr}\left(1 \Omega^{m-1} 0\right)+\operatorname{Pr}\left(0 \Omega^{m-1} 1\right)+\operatorname{Pr}\left(1 \Omega^{m-1} 1\right)=1$

and we express the ACF of a binary stationary process as

$$
R[m]=\operatorname{Pr}\left(1 \Omega^{m-1} 1\right)=1+\operatorname{Pr}\left(0 \Omega^{m-1} 0\right)-2 \operatorname{Pr}(0) .
$$

For the packet-error process of the DC model, $\operatorname{Pr}(0)=$ $\operatorname{Pr}\left(\beta_{k}=0\right)=P_{\mathrm{DC}}\left(0^{b}\right) \quad$ and $\operatorname{Pr}\left(0 \Omega^{m-1} 0\right)=\operatorname{Pr}\left(\beta_{k}=0\right.$, $\left.\beta_{k+m}=0\right)$ are obtained from $P_{\mathrm{DC}}\left(0^{2 b}\right)$, with the $(i, j)$ th entry of the $2 b \times 2 b$ normalized covariance matrix modified to $J_{0}\left(2 \pi f_{D}\left|i_{1}-j_{1}\right| T\right)$, where

$$
j_{1}= \begin{cases}j+(m-1) b, & \text { if } j \geq b+1 \\ j, & \text { if } j<b+1 .\end{cases}
$$

$$
L^{(M)}=\frac{\prod_{j=0}^{M-1-d_{1}^{M}}\left[j \frac{\varepsilon}{M-1+\alpha}+(1-\varepsilon)(1-p)\right] \prod_{j=0}^{d_{1}^{M}-1}\left[j \frac{\varepsilon}{M-1+\alpha}+(1-\varepsilon) p\right]}{\prod_{j=0}^{M-1}\left[1-(\alpha+j) \frac{\varepsilon}{M-1+\alpha}\right]}
$$

$$
R_{\mathrm{QBC}}[m]= \begin{cases}p, \frac{\varepsilon}{\frac{M-1+\alpha}{1-\frac{M-2+a l p h a}{M-\alpha} \varepsilon} p,} & \text { if } m=0 \\ (1-\varepsilon) p^{2}+\frac{\varepsilon}{M-1+\alpha}\left(\sum_{m-M+1}^{m-1} R_{\mathrm{QBC}}[i]+\alpha R_{\mathrm{QBC}}[m-M]\right), & \text { if } 1 \leq m \leq M-1 \\ & \text { if } m \geq M-1\end{cases}
$$


A similar definition holds for $i_{1}$. We denote $\operatorname{Pr}\left(\beta_{k}=0\right.$, $\left.\beta_{k+m}=0\right) \triangleq P_{\mathrm{DC}}^{\prime}\left(0^{2 b}\right)$. Thus, the ACF for the packet-error process of the DC model is expressed from (10) as

$$
R_{\beta}[m]=1+P_{\mathrm{DC}}^{\prime}\left(0^{2 b}\right)-2 P_{\mathrm{DC}}\left(0^{b}\right) .
$$

Substituting (10) evaluated at $m=1$ into (5) yields an alternative expression for the correlation coefficient of a binary stationary process

$$
\text { Cor }=\frac{\operatorname{Pr}(00)-\operatorname{Pr}^{2}(0)}{\operatorname{Pr}(0)(1-\operatorname{Pr}(0))}
$$

The correlation coefficient of the packet-error process of the DC model, which was denoted by $\mathrm{Cor}_{\beta}$, is thus expressed from (12) as

$$
\operatorname{Cor}_{\beta}=\frac{P_{\mathrm{DC}}\left(0^{2 b}\right)-P_{\mathrm{DC}}^{2}\left(0^{b}\right)}{P_{\mathrm{DC}}\left(0^{b}\right)\left(1-P_{\mathrm{DC}}\left(0^{b}\right)\right)}
$$

\section{B. Approximated Probability of the Packet-Error Process Under Slow Fading}

An approximation for $\operatorname{Pr}\left(\beta_{k}=1\right)$ and $R[m]$ for the packeterror process of the DC model with Rayleigh fading was derived in [4] under the assumption that the fading process is constant within a packet but varying from packet to packet according to Clarke's model. These approximations were applied in [4] to derive BAMNC models for the DC model at the packet level under slow-fading conditions. We herein generalize the results obtained in [4] by developing an expression for the probability of a packet-error sequence of length $n$ for the DC model with Rician fading under the same assumptions to parametrize higher order QBC models, as may be required in a slow-fading environment.

The probability of the packet error sequence for the DC model under slow-fading conditions is given by (the detailed derivation is provided in Appendix I)

$$
\begin{aligned}
\operatorname{Pr} & \left(\beta_{1}=\ell_{1}, \beta_{2}=\ell_{2}, \ldots, \beta_{n}=\ell_{n}\right) \\
= & \sum_{k_{1}=\ell_{1}}^{b} \cdots \sum_{k_{n}=\ell_{n}}^{b} \prod_{i=1}^{n}(-1)^{k_{i}+\ell_{i}}\left(\begin{array}{c}
b \\
k_{i}
\end{array}\right)\left(\frac{1}{2}\right)^{k_{i}} \\
& \times \frac{\exp \left\{-\frac{E_{s}}{N_{0}} K_{R} \mathbf{1}^{T} \mathbf{F}\left(\left(K_{R}+1\right) \mathbf{I}+\frac{E_{s}}{N_{0}} \overline{\mathbf{C}} \mathbf{F}\right)^{-1} \mathbf{1}\right\}}{\operatorname{det}\left(\mathbf{I}+\frac{E_{s}}{N_{0}}\left(1+K_{R}\right)^{-1} \overline{\mathbf{C}} \mathbf{F}\right)}
\end{aligned}
$$

where matrix $\mathbf{F}=\operatorname{diag}\left(k_{1} / 2, \ldots, k_{n} / 2\right)$, and the $(i, j)$ th entry of $\overline{\mathbf{C}}$ is $J_{0}\left(2 \pi f_{D}|i-j| b T\right), 1 \leq i, j \leq n$. In particular, two special cases of (14) are considered next, i.e.,

$$
\begin{aligned}
\operatorname{Pr}(1)= & \sum_{k=1}^{b}(-1)^{k+1}\left(\begin{array}{l}
b \\
k
\end{array}\right)\left(\frac{1}{2}\right)^{k} \frac{1+K_{R}}{1+K_{R}+k \frac{E_{s}}{2 N_{0}}} \\
& \times e^{-\frac{K_{R} E_{s}}{2 N_{0}} \frac{k}{1+K_{R}+k \frac{E_{s}}{2 N_{0}}}}
\end{aligned}
$$

$$
\begin{aligned}
\operatorname{Pr}(11)= & \sum_{k_{1}=1}^{b} \sum_{k_{2}=1}^{b}(-1)^{k_{1}+k_{2}}\left(\begin{array}{l}
b \\
k_{1}
\end{array}\right)\left(\begin{array}{l}
b \\
k_{2}
\end{array}\right)\left(\frac{1}{2}\right)^{k_{1}}\left(\frac{1}{2}\right)^{k_{2}} \frac{\left(1+K_{R}\right)^{2}}{D} \\
& \times e^{-\frac{K_{R} E_{s}}{2 N_{0}} \frac{\left(1+K_{R}+\frac{E_{s} k_{2}}{2 N_{0}}\right) k_{1}-2 k_{1} k_{2} \frac{E_{s}}{N_{0}} \rho+\left(1+K_{R}+\frac{E_{s} k_{1}}{2 N_{0}}\right) k_{2}}{D}}
\end{aligned}
$$

where $\rho=J_{0}\left(2 \pi f_{D} T\right)$, and

$D=\left(1+K_{R}\right)^{2}+\frac{E_{s}}{2 N_{0}}\left(1+K_{R}\right)\left(k_{1}+k_{2}\right)+\left(\frac{E_{s}}{2 N_{0}}\right)^{2}\left(1-\rho^{2}\right) k_{1} k_{2}$.

The next section considers the problem of fitting the discretized Rayleigh and Rician DC models at the packet level using QBC models (including the BAMNC model).

\section{QBC Model Parameter Estimation}

\section{A. BAMNC Model}

We apply the formulas derived in the previous section to find the parameters of the BAMNC that best fit the DC model at the packet level. In this section, the accuracy of the BAMNC is measured in terms of the ACF criterion.

Given a DC model with fixed $E_{s} / N_{0}, f_{D} T$, and $K_{R}$, we first calculate $P_{\mathrm{DC}}\left(0^{b}\right)$ and $P_{\mathrm{DC}}\left(0^{2 b}\right)$ using (2) and then the probability of packet error $\mathrm{PPE}_{\beta}$ and the correlation coefficient $\operatorname{Cor}_{\beta}$ using (9) and (13), respectively. The two parameters of the BAMNC are obtained by setting $p=\mathrm{PPE}_{\beta}$ and $\varepsilon=\mathrm{Cor}_{\beta}$. We denote by BAMNC under slow fading (BAMNC-SF) a BAMNC whose parameters $p$ and $\varepsilon$ are derived from (15) and (16), respectively.

Fig. 2 compares (at the packet level) the ACF of the Rayleigh DC model with the ACF of the BAMNC and BAMNC-SF models that are fitted to the DC model. The DC model has parameters $E_{s} / N_{0}=20 \mathrm{~dB}$ and $K_{R}=-\infty \mathrm{dB}$ (Rayleigh fading), with $f_{D} T=0.005$ in Fig. 2(a) and $f_{D} T=0.001$ in Fig. 2(b). The packets are of length 8 . The ACF of the DC model at the packet level is calculated using (11), and the estimated parameters of the BAMNC (e.g., $p=0.0419, \varepsilon=0.46$ for $f_{D} T=0.005$ and $p=0.0395, \varepsilon=0.6518$ for $f_{D} T=0.001$ ) and BAMNC-SF (e.g., $p=0.0394, \varepsilon=0.453$ for $f_{D} T=$ 0.005 and $p=0.0394, \varepsilon=0.6522$ for $f_{D} T=0.001$ ) are substituted into (8) to get the ACF of the BAMNC models. We observe a good ACF agreement between the BAMNC and DC models when $f_{D} T$ is greater than (curves not shown) or equal to 0.005. For $f_{D} T=0.005$, the ACF curves for these two models coincide. The BAMNC-SF may be considered acceptable for $f_{D} T=0.005$, but this will further be investigated in the next section. When $f_{D} T=0.001$, the ACF curves for the BAMNC and BAMNC-SF are identical, but these curves exhibit greater discrepancies when compared with those of the DC model, which indicates that higher order QBC models are required for modeling DC models at the packet level with slowly varying fading channels.

\section{B. $Q B C$ Model With $M>1$}

We next employ (14) to find the probability of all packeterror sequences of length $M+1$ and use these probabilities 


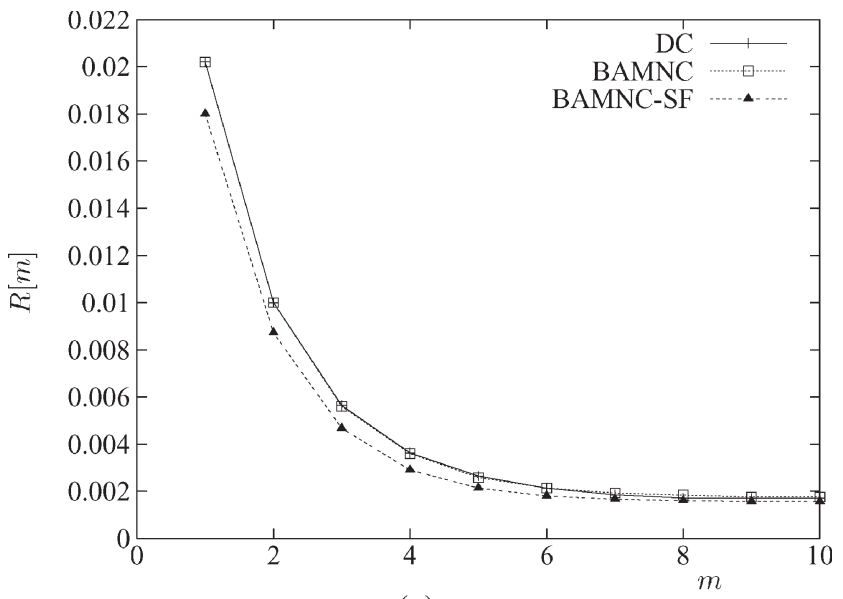

(a)

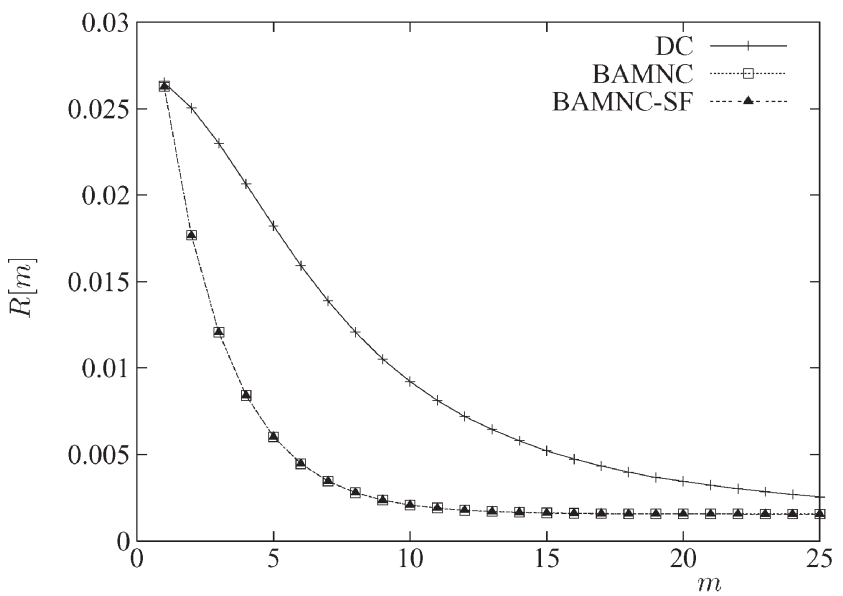

(b)

Fig. 2. Comparison of the ACFs of the Rayleigh DC and the BAMNC for $E_{s} / N_{0}=20 \mathrm{~dB}, K_{R}=-\infty \mathrm{dB}$, and (a) $f_{D} T=0.005$ or (b) $f_{D} T=$ 0.001 . The packets are of length 8 .

to parametrize the $M$ th-order $\mathrm{QBC}$ model $(M>1)$ at the packet level under slow-fading conditions. We denote these models as $M$-QBC-SF. This is achieved using the methodology proposed in [11] and [20], which selects the QBC parameters that minimize the Kullback-Leibler divergence rate between the DC and QBC packet error processes defined as

$$
D\left(P_{\beta} \| P_{\mathrm{QBC}}\right) \triangleq \lim _{n \rightarrow \infty} \frac{1}{n} \sum_{z^{n} \in\{0,1\}^{n}} P_{\beta}\left(z^{n}\right) \log _{2} \frac{P_{\beta}\left(z^{n}\right)}{P_{\mathrm{QBC}}\left(z^{n}\right)}
$$

for identical packet error rates and noise correlation coefficients. ${ }^{1}$ Expressions for $P_{\mathrm{QBC}}\left(z^{n}\right)$ in terms of the QBC parameters are given in (3) and (4), and $P_{\beta}\left(z^{n}\right) \triangleq$

\footnotetext{
${ }^{1}$ Note that, for the same BER, matching the correlation coefficient between the two binary channels is equivalent to matching their probabilities of two consecutive errors. Ideally, when matching one channel with memory to another, one would want to match their $n$-dimensional distributions for every $n$. However, as this is infeasible in general, a natural way to fit the two binary channels is to match their lower order statistics, such as their BER (for $n=1$ ) and their probabilities of two consecutive errors (for $n=2$ ), and minimize the Kullback-Leibler divergence rate between the channels' $n$-fold error distributions since one would desire identical statistical behaviors on both channels for large block lengths (as large block lengths are required to achieve reliable communication by the channel coding theorem).
}

$\operatorname{Pr}\left(\beta_{1}=z_{1}, \beta_{2}=z_{2}, \ldots, \beta_{n}=z_{n}\right)$. It is known (e.g., [11] and [20]) that $D\left(P_{\beta} \| P_{\mathrm{QBC}}\right)$ exists and is given by

$$
\begin{aligned}
D\left(P_{\beta} \| P_{\mathrm{QBC}}\right) & =-\lim _{n \rightarrow \infty} \frac{1}{n} H\left(P_{\beta}\left(z^{n}\right)\right) \\
& -\sum_{z^{M+1}} P_{\beta}\left(z^{M+1}\right)\left[\log _{2} P_{\mathrm{QBC}}\left(z_{M+1} \mid z^{M}\right)\right]
\end{aligned}
$$

where $P_{\mathrm{QBC}}\left(z_{M+1} \mid z^{M}\right)$ is the $\mathrm{QBC}$ conditional probability of noise symbol $z_{M+1}$, given the previous $M$ symbols, which is evaluated using the QBC noise block probability (3) and (4). Let the probability in (15) be denoted by $\mathrm{PPE}_{\beta-\mathrm{SF}}$ and the noise correlation coefficient obtained by substituting (15) and (16) into (5) be denoted by $\mathrm{Cor}_{\beta-\mathrm{SF}}$. We then set $p=\mathrm{PPE}_{\beta-\mathrm{SF}}$ and $\mathrm{Cor}_{\mathrm{QBC}}=\mathrm{Cor}_{\beta-\mathrm{SF}}$. Parameter $\alpha$ is then expressed from (6) as

$$
\alpha=\frac{\varepsilon+\operatorname{Cor}_{\beta-\mathrm{SF}}(1-M)+(M-2) \varepsilon}{\operatorname{Cor}_{\beta-\mathrm{SF}}(1-\varepsilon)} .
$$

For a fixed $\operatorname{Cor}_{\mathrm{QBC}}>0$ and $M$, parameter $\alpha$ is a nondecreasing function of $\varepsilon$ and is nonnegative whenever $\varepsilon$ is in interval $\Delta$ given by

$$
\Delta=\left[\frac{\operatorname{Cor}_{\mathrm{QBC}}(M-1)}{1+\operatorname{Cor}_{\mathrm{QBC}}(M-2)}, 1\right] .
$$

For example, for $\operatorname{Cor}_{\mathrm{QBC}}=0.8$, then $\Delta=[0.8888,1]$ if $M=3$ and $\Delta=[0.9412,1]$ if $M=5$. Parameter $\varepsilon \in \Delta$ is selected to minimize (18) or, equivalently, to maximize

$$
\sum_{z^{M+1}} P_{\beta}\left(z^{M+1}\right)\left[\log _{2} P_{\mathrm{DC}}\left(z_{M+1} \mid z^{M}\right)\right] .
$$

As a result of the optimization procedure, the QBC-SF parameters for selected values of $E_{s} / N_{0}$, Rayleigh fading $\left(K_{R}=\right.$ $-\infty \mathrm{dB})$, packets of length $b=8$, and $f_{D} T=0.001(M=2$ and $M=3)$ and $f_{D} T=0.0005(M=4)$ are given in Table I. The ACFs of the DC and $M$-QBC-SF models, for $M=1,2$, and 3, are compared in Fig. 3 for the DC model with Rayleigh fading, $E_{s} / N_{0}=20 \mathrm{~dB}$, and $f_{D} T=0.001$. A significant modeling improvement is obtained over the BAMNC models [compared with Fig. 2(b)] when $M>1$. Similar results were observed for packet lengths of $b=6$ and 10 .

\section{Probability of Codeword ERror}

While the ACF has empirically been shown to be an effective criterion for measuring the similarity between channels with memory (e.g., two channels with closely similar ACFs also agree in terms of capacity or error exponent) [10], [11], [20], [33]-[35], it may not necessarily indicate that two channels with matching ACFs will experience identical PCE performances when subjected to the same code (under the same decoding scheme). Thus, to further investigate and validate the accuracy of the QBC models in approximating the packet-error process $\left\{\beta_{k}\right\}_{k=1}^{\infty}$ of the DC model, we analyze the PCE performance of $(n, k) \mathrm{RS}$ codes over these models. Our numerical results will show that it is not always the case that the agreement between the DC and the QBC in terms of ACF also holds 
TABLE I

Good $M$-QBC-SF Models for Rayleigh Fading $\left(K_{R}=-\infty \mathrm{dB}\right)$. The Packets Are of Length $b=8$

\begin{tabular}{|c|c|c|c|}
\hline \hline$E_{s} / N_{0}$ & $f_{D} T=0.001(M=2)$ & $f_{D} T=0.001(M=3)$ & $f_{D} T=0.0005(M=4)$ \\
\hline \multirow{2}{*}{$15 \mathrm{~dB}$} & $p=0.1176$ & $p=0.1176$ & $p=0.1176$ \\
& $\varepsilon=0.7773$ & $\varepsilon=0.8339$ & $\varepsilon=0.8773$ \\
& $\alpha=0.9018$ & $\alpha=0.7354$ & $\alpha=0.8359$ \\
\hline \multirow{2}{*}{$20 \mathrm{~dB}$} & $p=0.0394$ & $p=0.0394$ & $p=0.0394$ \\
& $\varepsilon=0.7672$ & $\varepsilon=0.8215$ & $\varepsilon=0.8774$ \\
& $\alpha=0.7574$ & $\alpha=0.454$ & $\alpha=0.4665$ \\
\hline \multirow{2}{*}{$25 \mathrm{~dB}$} & $p=0.0127$ & $\varepsilon=0.0127$ & $p=0.0127$ \\
& $\varepsilon=0.7164$ & $\alpha=0.1689$ & $\varepsilon=0.8643$ \\
& $\alpha=0.4874$ & \multicolumn{3}{|c|}{} \\
\hline
\end{tabular}

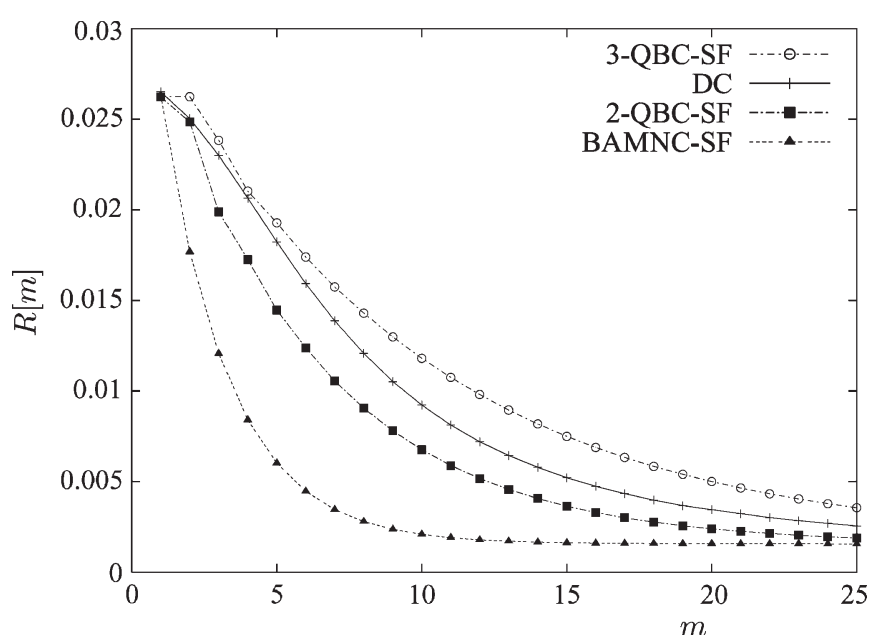

Fig. 3. Comparison of the ACFs of the Rayleigh DC $\left(K_{R}=-\infty \mathrm{dB}\right)$ and the $M$-QBC-SF $(M=1,2$, and 3$)$ for $E_{s} / N_{0}=20 \mathrm{~dB}$ and $f_{D} T=0.001$. The packets are of length $b=8$.

in terms of the PCE performance. ${ }^{2}$ It is nevertheless worth pointing out that, in all our results, we did note that the ACF was still useful in predicting the memory order of the QBC that best fits the DC in terms of PCE.

Under bounded distance decoding, the PCE for RS codes over binary FSMC models is obtained using $P_{s}(m, n)$, which is the probability that $m$-packet (symbols) errors occur in a block of $n$ packets [26]-[28], where each packet is given by sequence $E_{(k-1) b+1}, \ldots, E_{k b}, k \geq 1$. However, determining $P_{s}(m, n)$ can be quite complicated, particularly when $b \gg 1$. Since the binary FSMC model at the packet level generates a binary process $\left\{\beta_{k}\right\}_{k=1}^{\infty}$, where $\beta_{k}=1$ indicates an RS symbol error, the simpler expressions for the probability that $m$-bit errors occur in a block with a length of $n$ bits, i.e., $P(m, n)$, that were developed to study the performance of binary codes over binary FSMC models at the bit level can thus be applied to study the performance of RS codes over these models at the packet level. Thus, for a $t$ error-correcting RS code over an FSMC model at the packet level, the PCE under bounded distance decoding is given by

$$
\mathrm{PCE}=1-\sum_{m=0}^{t} P(m, n)=1-P_{c}
$$

where $P_{c}$ is the probability of correct decoding. Henceforth, we concentrate on the calculation of $P(m, n)$. Recursive formulas

${ }^{2}$ See the next section, and compare Fig. 2(b) with Fig. 4 for the BAMNC and Fig. 3 with Fig. 6 for the 2-QBC-SF. for $P(m, n)$ have been derived for some classes of FSMC models in [9] and [28]-[30].

\section{A. Closed-Form Expression of $P(m, n)$ for the BAMNC}

A closed-form formula for $P(m, n)$ for the BAMNC ${ }^{3}$ is derived in [9, eq. (39)]. A much simpler closed-form expression is derived in this section, following the approach proposed in [29] that expresses $P(m, n)$ in terms of a coefficient in a formal power series. This is outlined next.

Given indeterminates $s$ and $z$, we define the formal power series $P(s, z)=\sum_{n=0}^{\infty} \sum_{m=0}^{n} P(m, n) s^{m} z^{n}$. Thus, $P(m, n)$ for a binary FSMC is written as the coefficient of $s^{m} z^{n}$ in $P(s, z)$, which is denoted by $P(m, n)=\left[s^{m} z^{n}\right] P(s, z)$. An expression for $P(s, z)$ in terms of matrices $\mathbf{P}(0)$ and $\mathbf{P}(1)$ that specifies a binary FSMC is given in [29]

$$
P(s, z) \triangleq \mathbf{\Pi}^{T}[\mathbf{I}-z\{\mathbf{P}(0)+s \mathbf{P}(1)\}]^{-1} \mathbf{1} .
$$

The generating series $P(s, z)$ for the BAMNC model is given by substituting matrices $\mathbf{P}(0)$ and $\mathbf{P}(1)$ obtained from (7) and state stationary vector $\boldsymbol{\Pi}^{T}=[1-p, p]$ into (20). Thus

$$
P(s, z)=\frac{1-\varepsilon(1-p) s z-\varepsilon p z}{\left.1-(p+\varepsilon(1-p)) s z-(\varepsilon p+(1-p)) z+\varepsilon s z^{2}\right]} .
$$

Following the partial fraction technique described in [29, App.] to extract the coefficient of $P(s, z)$, we arrive at (the detailed derivation is provided in Appendix II)

$$
\begin{gathered}
P(m, n)=x^{m} y^{n-m}\left\{\sum_{\ell=0}^{m} \frac{\left[\prod_{j-\ell+1}^{M-1+\ell}(n-j)\right]}{(m-\ell) ! \ell !}\left(\frac{-\varepsilon}{x y}\right)^{\ell}\right. \\
\left.\times\left[\frac{(1-p)(n-\ell)}{y}-\frac{\varepsilon(1-p)(m-\ell)}{x}+\frac{\varepsilon p m}{y}\right]\right\}
\end{gathered}
$$

for $n \geq 2 m+1$, where

$$
\begin{cases}\prod_{j=a}^{b}(\cdot)=1, & \text { if } a=b+1 \\ \prod_{j=a}^{b}(\cdot)=\frac{1}{\prod_{j=b+1}^{a-1}(\cdot)}, & \text { if } a>b+1 .\end{cases}
$$

${ }^{3}$ The simplified Gilbert channel studied in [9] is exactly identical to the BAMNC considered here. 
TABLE II

PCE ALGORITHM DESCRIPTION

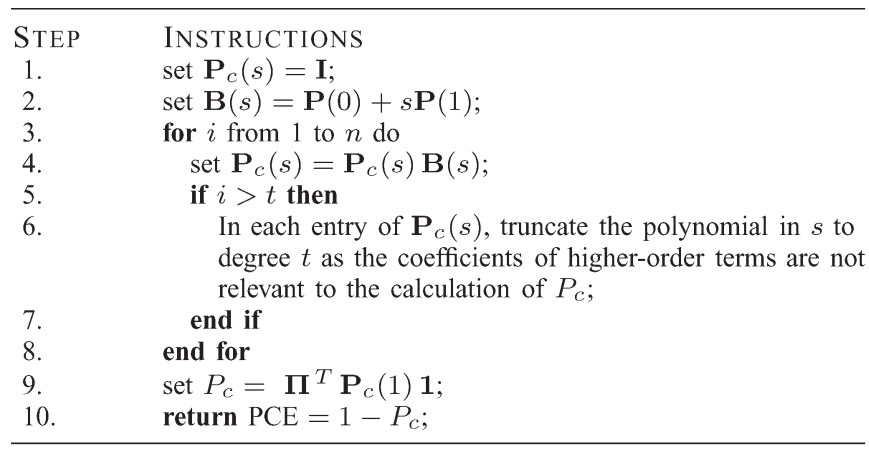

Equations (19) and (21) are used to calculate the PCE for the BAMNC. We now consider two special cases of (21). When $\varepsilon=0$, we obtain $x=p$ and $y=1-p$, and the nonzero term in the summation (corresponding to $\ell=0$ ) becomes $\left(\begin{array}{l}n \\ m\end{array}\right)$. When $m=0$ and $n>0$, we obtain from (22)

$$
P(0, n)=y^{n} \frac{1}{n}\left[\frac{(1-p) n}{y}\right]=((1-p)+\varepsilon p)^{n-1}(1-p) .
$$

Equations (19) and (21) are used to calculate the PCE for the BAMNC. Note that the closed-form expression for $P(m, n)$ in (21) is significantly simpler than that derived in [9, eq. (39)]. Furthermore, if we are to model the system at the bit level, we would need to determine $P_{s}(m, n)$, but the latter admits a recursive expression for the BAMNC [28], unlike the simple closed-form formula in (21).

\section{B. $P(m, n)$ Algorithm for the $Q B C$ With $M>1$}

Given a QBC with $M>1$, the derivation of a closed-form expression for $P(m, n)$ is tedious. Let $\mathbf{B}(s)=\mathbf{P}(0)+s \mathbf{P}(1)$. The method proposed in [36], which is suitable for FSMC models with a small number of states, calculates $P(m, n)$ as

$$
P(m, n)=\left[s^{m}\right] \boldsymbol{\Pi}^{T} \mathbf{B}^{n}(s) \mathbf{1}, \quad \text { for } 0 \leq m \leq n .
$$

The calculation of $\mathbf{B}^{n}(s)$ is a complex task, especially for high-order FSMC models, and $\mathbf{B}^{n}(s)$ contains much more information that is needed to calculate $P_{c}$ in (19), i.e., $P(m, n)$ for $0 \leq m \leq t$.

We propose in Table II a matrix-based iterative algorithm that is valid for any FSMC model. The $n$th power of $\mathbf{B}(s)$ can iteratively be calculated as $\mathbf{B}^{n}(s)=\mathbf{B}^{n-1}(s) \mathbf{B}(s)$ (for loop in step 3$)$. The $(i, j)$ th entry of $\mathbf{B}^{\ell}(s)$ is a polynomial of the form $\sum_{j=0}^{\ell} p_{i, j}^{\ell, j} s^{j}$, where $p_{i, j}^{\ell, j}$ is the probability that the FSMC, starting from state $i$ and ending at state $j$, generates an error sequence of length $\ell$ with Hamming weight $j$. These probabilities will never be used in the calculation of $P_{c}$ when $j>t$ and can be discarded at each iteration of the for loop. (This is performed in step 6.) Thus, the algorithm discards at each iteration the probability that the FSMC, starting from state $i$ and ending at state $j$, generates an error sequence of length $\ell>t$ with a Hamming weight that is larger than $t$. As a result, the computational complexity of the algorithm is reduced. The $(i, j)$ th entry of $\mathbf{P}_{c}(1)$ after step 6 is the probability that the

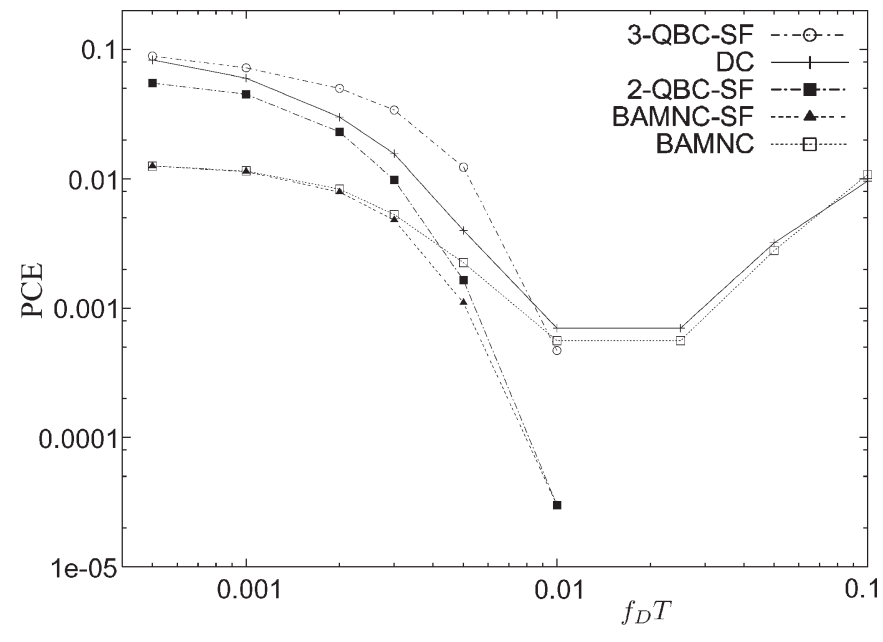

Fig. 4. PCE versus $f_{D} T$ for the $(255,197)$ RS code $(b=8, t=29)$ over the BAMNC and $M$-QBC-SF $(M=1,2,3)$. A DC model with Rayleigh fading $\left(K_{R}=-\infty \mathrm{dB}\right)$ and $E_{s} / N_{0}=20 \mathrm{~dB}$ was used.

FSMC, starting from state $i$ and ending at state $j$, generates an error sequence with a Hamming weight that is less than or equal to $t$. Thus, $P_{c}$ is calculated in accordance to (19). It is worth noting that, due to the structure of the $\mathrm{QBC}$, the $2^{M} \times 2^{M}$ matrix $\mathbf{B}(s)$ in step 2 is sparse, having only two nonzero entries in each row and each column, which facilitates the computation of $\mathbf{P}_{c}(s)$ in the main loop of the algorithm.

\section{NumericAl PCE RESUlts}

This section presents the PCE curves when an RS code is transmitted over the DC model and its QBC approximation at the packet level. For specific values of the DC model parameters $\left(E_{s} / N_{0}, f_{D} T\right.$, and $K_{R}$ ), we first calculate the QBC parameters according to the procedure described in Section IV and then use the development in Section V to numerically determine the PCE for the QBC model. To verify the effectiveness of a particular QBC model, the PCE results for RS codes over the DC model (as described in Fig. 1) are obtained by simulations (PCE simulations over the binary BFSK modulated harddecision demodulated fading channels). In this case, the binary representation of each RS codeword is transmitted across the binary DC model. Specific $M$-QBC models that match the simulated results for several codes and DC model parameters are discussed in the succeeding sections.

\section{A. Rayleigh Fading}

Fig. 4 shows PCE versus $f_{D} T$ for the $(255,197)$ RS code ( $b=8, t=29$ symbols) over the BAMNC and $M$-QBC-SF ( $M=1,2$, and 3 ), which approximates the DC model with Rayleigh fading $\left(K_{R}=-\infty \mathrm{dB}\right)$ for $E_{s} / N_{0}=20 \mathrm{~dB}$. Simulation results for the DC model are labeled as DC in the figures of this section. We observe that the BAMNC-SF is not adequate for modeling the considered DC models. The PCE curves of the DC model and the 2-QBC-SF and 3-QBC-SF match quite well when $f_{D} T \leq 0.002$. In particular, the PCE values of the DC model and the 3-QBC-SF are almost identical when $f_{D} T \leq 0.001$. (We observed in Fig. 3 a good agreement in ACF 


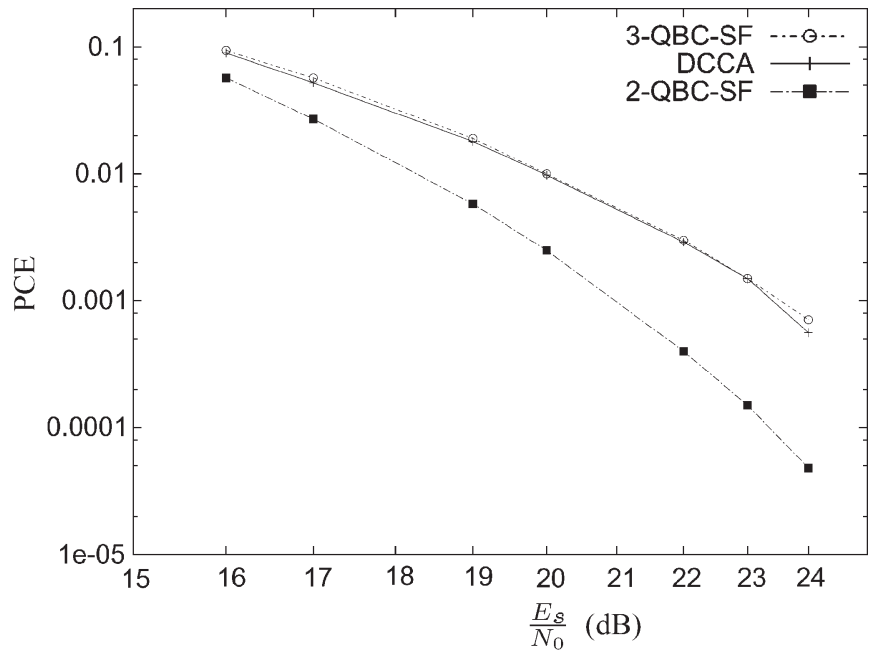

Fig. 5. PCE versus $E_{s} / N_{0}$ for the $(255,155)$ RS code $(b=8, t=50)$ over the $M$-QBC-SF ( $M=2$ and 3$)$. A DC model with Rayleigh fading $\left(K_{R}=\right.$ $-\infty \mathrm{dB}$ ) and $f_{D} T=0.001$ was used.

between the DC model and its 3-QBC-SF approximation when $f_{D} T=0.001$.) The BAMNC is reasonably accurate for fast and medium fading rates, i.e., $f_{D} T \geq 0.01$. Surprisingly, the accuracy of the BAMNC shown in Fig. 2(b) for $f_{D} T=0.005$ (according to the ACF criterion) is not validated in Fig. 4 (under the PCE criterion).

Fig. 5 shows the PCE curves versus $E_{s} / N_{0}$ for the (255, $155) \mathrm{RS}$ code $(b=8, t=50$ symbols) over the $M$-QBC-SF $(M=2$ and 3$)$ for the DC model with Rayleigh fading $\left(K_{R}=\right.$ $-\infty \mathrm{dB}$ ) for $f_{D} T=0.001$. We clearly note that 3-QBC-SF is an accurate model for the Rayleigh DC model with $f_{D} T=$ 0.001 for a broad range of $E_{s} / N_{0}$. The robustness of this model is also verified for RS codes with different values of $t$, as shown in Fig. 6. It is seen from this figure that the 2-QBC-SF is good for high rate codes $(t \leq 25)$; in this case, further PCE improvement may be achieved by incorporating symbol interleaving into the communication system. We observe that the discrepancies between the 2-QBC-SF and the 3-QBC-SF increases when $E_{s} / N_{0}$ and $t$ increase; for low rate codes $(t>$ $25)$, the 2-QBC-SF does not match the DC in terms of PCE in spite of a relatively good agreement between the two channels in terms of ACF (see Fig. 3). As shown in Fig. 7, the 4-QBC-SF provides accurate results in a slower varying fading channel with $f_{D} T=0.0005$ over a broad range of $E_{s} / N_{0}$. Thus, the QBC-SF parameters in Table I are accurate when $M=3$ and $M=4$, whereas the case $M=2$ is valid in some special cases.

We conclude from the figures shown in this section that, for a packet of length 8 , the BAMNC is an accurate model for $f_{D} T>0.01$ and that, for $f_{D} T \leq 0.001$, the $M$-QBC-SF with $M \geq 3$ is adequate. The same trend is observed for other values of $b$, as shown in Fig. 8, where the PCE curves are plotted versus $t$ for an RS code with parameters $n=63$ and $b=6$. In the scenario of medium varying fading $0.01<f_{D} T<0.001$, neither the BAMNC nor the $M$-QBC-SF fits the DC model. We also observe from this figure $\left(f_{D} T=0.005\right)$ that the $2-\mathrm{QBC}$ is capable of approximating the DC model in this range.

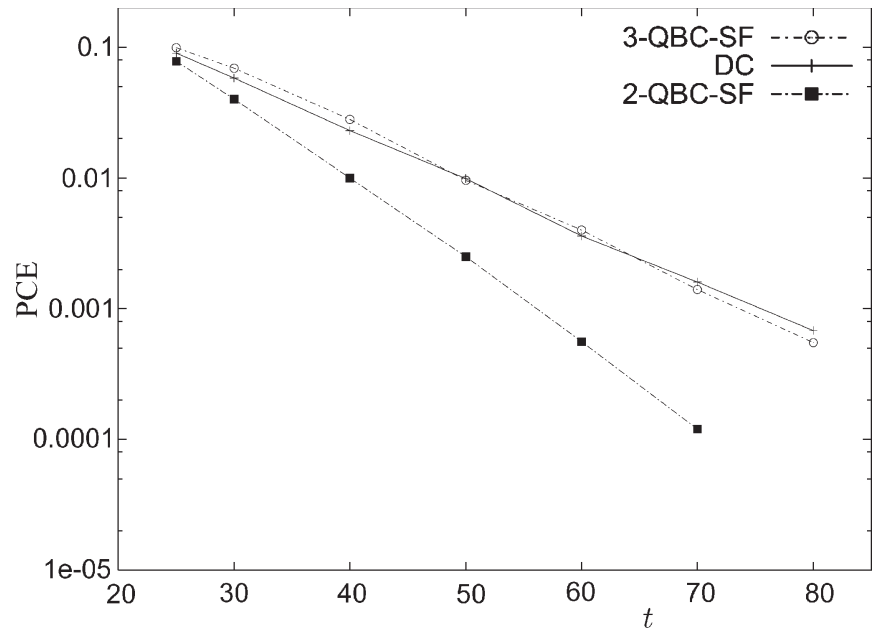

Fig. 6. PCE versus $t$ for the RS code $(n=255, b=8)$ over the $M$-QBC-SF $\left(M=2\right.$ and 3). A DC model with Rayleigh fading $\left(K_{R}=-\infty \mathrm{dB}\right), f_{D} T=$ 0.001 , and $E_{s} / N_{0}=20 \mathrm{~dB}$ was used.

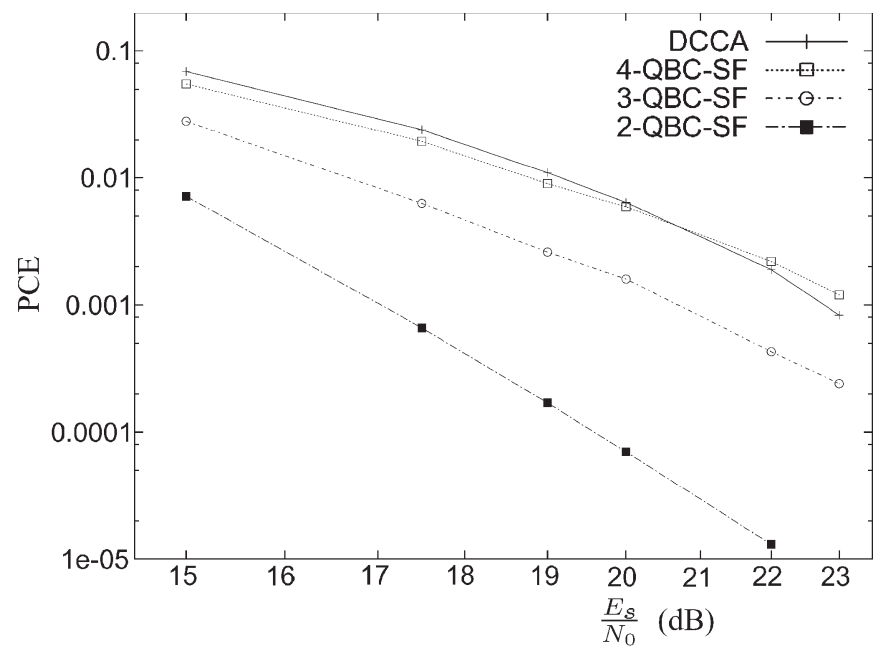

Fig. 7. PCE versus $E_{s} / N_{0}$ for the $(255,95) \mathrm{RS}$ code $(b=8, t=80)$ over the $M$-QBC-SF ( $M=2,3$, and 4$)$. A DC model with Rayleigh fading $\left(K_{R}=\right.$ $-\infty \mathrm{dB})$ and $f_{D} T=0.0005$ was used.

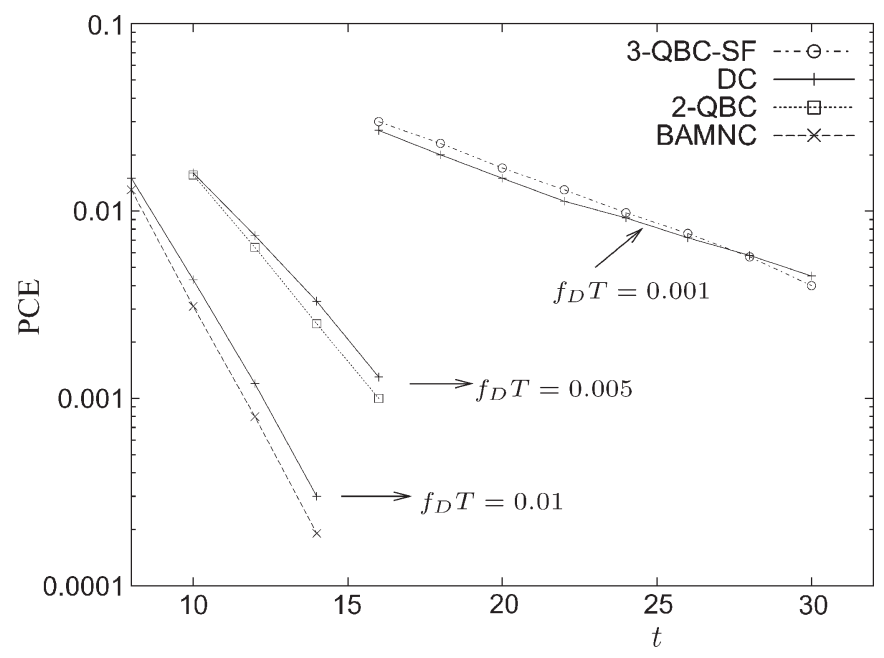

Fig. 8. PCE versus $t$ for the RS code $(n=63, b=6)$ over the 3-QBC-SF $\left(f_{D} T=0.001\right)$, the 2-QBC $\left(f_{D} T=0.005\right)$, and the BAMNC $\left(f_{D} T=\right.$ $0.01)$ for Rayleigh fading $\left(K_{R}=-\infty\right)$ and $E_{s} / N_{0}=20 \mathrm{~dB}$. 


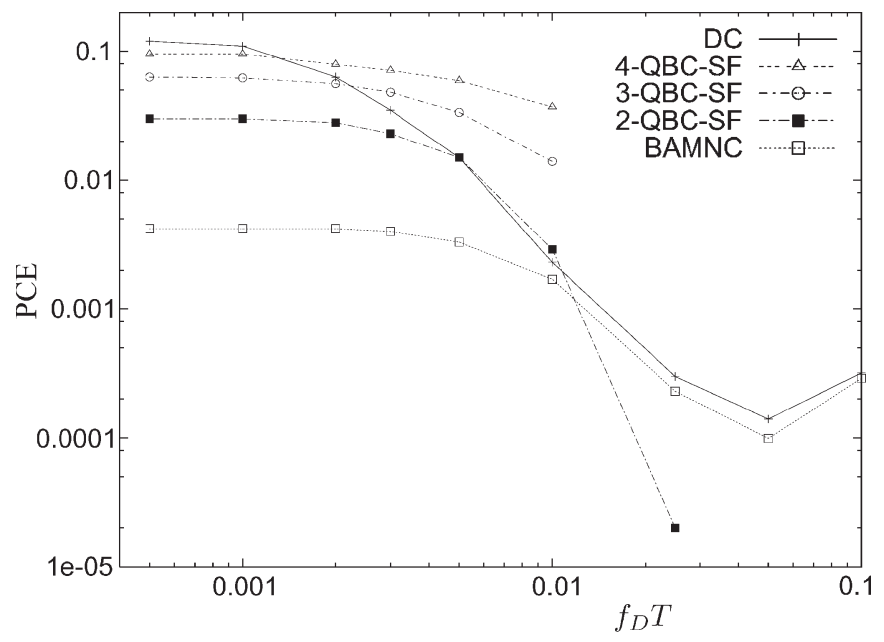

Fig. 9. PCE versus $f_{D} T$ for the $(255,197)$ RS code $(b=8, t=29)$ over the BAMNC and $M$-QBC-SF ( $M=2,3$, and 4). A DC model with Rician fading, $K_{R}=5 \mathrm{~dB}$, and $E_{s} / N_{0}=15 \mathrm{~dB}$ was used.

A similar behavior was observed for $b=10$ (curves not shown). The Rician DC channel is considered next.

\section{B. Rician Fading}

Fig. 9 shows PCE versus $f_{D} T$ for the $(255,197)$ RS code ( $b=8, t=29$ symbols) over the BAMNC and $M$-QBC-SF ( $M=2,3$, and 4 ) for the DC model with Rician fading, $K_{R}=$ $5 \mathrm{~dB}$, and $E_{s} / N_{0}=15 \mathrm{~dB}$. The BAMNC is accurate in the same range of $f_{D} T$ observed in the Rayleigh case $\left(f_{D} T>\right.$ 0.01 ), whereas the 4-QBC-SF is a good model when $f_{D} T<$ 0.001 . We also observe that the PCE is smallest for $f_{D} T \approx 0.05$ (see Figs. 4 and 9) and increases when $f_{D} T$ increases beyond 0.05 . This is because, for fast fading, the channel behaves like a memoryless channel, making the errors occur at random within a codeword; hence, the RS code does not correct enough error symbols (as it is better suited for correcting errors that occur in bursts). When $f_{D} T$ decreases, the error bits become more concentrated within bursts and affect fewer symbols of a codeword. Therefore, for the used RS codes, short bursts yield the best performance. When $f_{D} T=0.001$ and $E_{s} / N_{0}=$ $15 \mathrm{~dB}$, Fig. 10 shows that the 4-QBC-SF is valid for a broad range of values of $t$. In Fig. 11, the behavior of PCE versus $E_{s} / N_{0}$ is shown for the $(255,155) \mathrm{RS}$ code $(b=8, t=50)$ over the $M$-QBC-SF $(M=2,3,4)$. We remark that the 4-QBC-SF is adequate when $E_{s} / N_{0}<17 \mathrm{~dB}$. This limiting value of $E_{s} / N_{0}$ is increased to $20 \mathrm{~dB}$ when $t=29$. Thus, the robustness of a given $M$-QBC-SF model for different values of $t$ and $E_{s} / N_{0}$ observed in the Rayleigh case is not observed for Rician fading.

The parameters of the QBC model at the bit level that fit the DC model considered in this work are found in [11]. For $E_{s} / N_{0}=15 \mathrm{~dB}$ and $f_{D} T=0.001$, the QBC at the bit level requires $M=20$ for Rayleigh fading $\left(K_{R}=-\infty \mathrm{dB}\right)$ and $M=18$ for Rician fading $\left(K_{R}=5 \mathrm{~dB}\right)$. These values of memory order are significantly larger than those found in this work $(M \leq 4)$. Therefore, whenever the performance of nonbinary codes is considered, significant modeling complex-

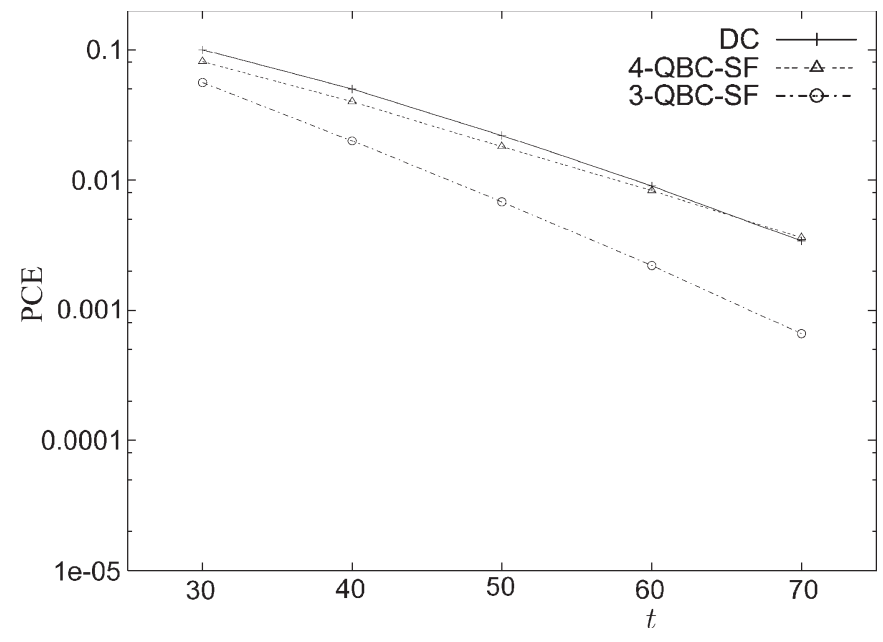

Fig. 10. PCE versus $t$ for the RS code $(n=255, b=8)$ over the $M$-QBC-SF ( $M=3$ and 4). A DC model with Rician fading, $K_{R}=5 \mathrm{~dB}, f_{D} T=0.001$, and $E_{s} / N_{0}=15 \mathrm{~dB}$ was used.

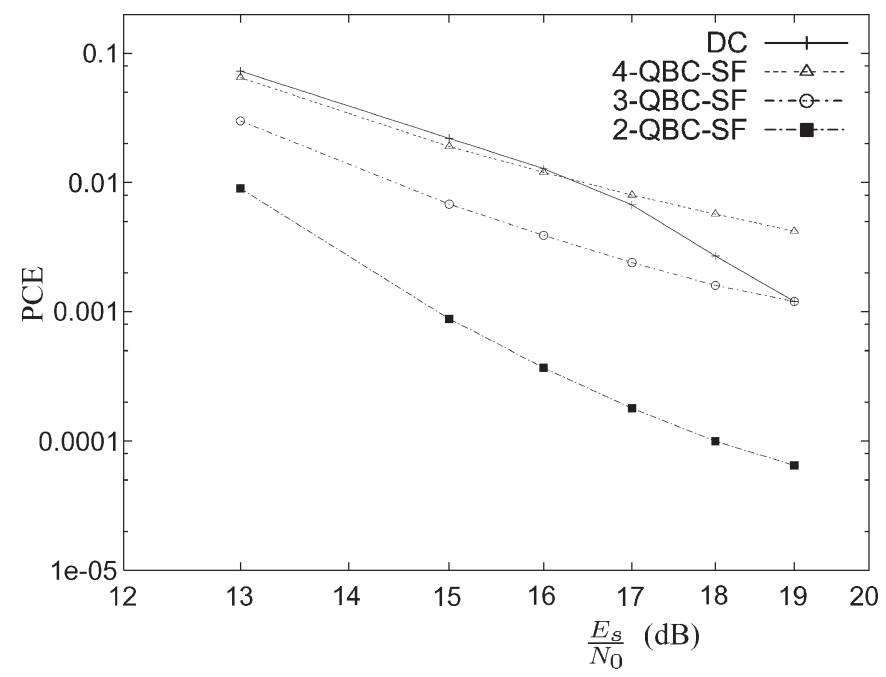

Fig. 11. PCE versus $E_{s} / N_{0}$ for the (255,155) RS code $(b=8, t=50)$ over the $M$-QBC-SF ( $M=2,3$, and 4$)$. A DC model with Rician fading, $K_{R}=$ $5 \mathrm{~dB}$, and $f_{D} T=0.001$ was used.

ity reduction is achieved by the QBC model at the packet level.

\section{CONCLUSION}

The majority of previous works on modeling the physical layer of a communication system using FSMC models can be divided into two categories: 1) modeling the information packet-error process from the input of the channel encoder to the output of the channel decoder and 2) modeling the bit error process of the DC model (from the input of the modulator to the output of the demodulator). This work models the packet (symbol) error process of the DC model via the QBC.

We developed the $M$ th-order Markovian QBC models at the packet (symbol) level for an RS-coded DC representing a hard-decision demodulated Rician fading channel. We denoted by $M$-QBC-SF a QBC model with memory $M$ parametrized with the probabilities of the DC model derived under the assumption that the fading process is constant within a packet. 
The ACF of the DC model at the packet level can theoretically be computed and can give an estimate of the memory order required by the QBC. However, since one of the main goals of a model is to generate a PCE, which is approximately the same as the PCE for the original channel, we evaluated the QBC models in terms of the PCE criterion. The comparison of the PCE obtained analytically (for the QBC model) and by simulation (for the DC model) revealed that the $M$-QBC-SF is a good approximation for the DC model when $f_{D} T \geq 0.001$. In particular, such QBC models with $M \leq 4$ were shown to well approximate the DC models with slow Rayleigh fading for a broad range of signal-to-noise ratios. In addition, the 4-QBC-SF is adequate for Rician DC models with $f_{D} T=0.001$. An interesting direction for future work is to use the QBC models developed in this paper to derive (block-by-block and iterative) decoding strategies (e.g., see [17]-[19] and [37]-[39]) for RS codes that can exploit channel memory.

\section{APPENDIX I}

DERIVATION OF (14)

Let $A_{k}=|\tilde{G}(k T)|$ be a constant fading amplitude within a packet. For a packet of length $b$, using the binomial theorem, the conditional packet error probability can be written as [4]

$$
\begin{aligned}
\operatorname{Pr}\left(\beta_{1}=1 \mid a_{1}\right) & \triangleq \operatorname{Pr}\left(\beta_{1}=1 \mid A_{1}=a_{1}\right) \\
& =1-\left[1-\operatorname{Pr}\left(E_{1}=1 \mid a_{1}\right)\right]^{b} \\
& =\sum_{k=1}^{b}(-1)^{k+1}\left(\begin{array}{l}
b \\
k
\end{array}\right)\left[\operatorname{Pr}\left(E_{1}=1 \mid a_{1}\right)\right]^{k} .
\end{aligned}
$$

On the other hand

$$
\operatorname{Pr}\left(\beta_{1}=0 \mid a_{1}\right)=\sum_{k=0}^{b}(-1)^{k}\left(\begin{array}{l}
b \\
k
\end{array}\right)\left[\operatorname{Pr}\left(E_{1}=1 \mid a_{1}\right)\right]^{k} .
$$

Combining (24) and (25), we get

$$
\operatorname{Pr}\left(\beta_{1}=\ell_{1} \mid a_{1}\right)=\sum_{k=\ell_{1}}^{b}(-1)^{k+\ell_{1}}\left(\begin{array}{l}
b \\
k
\end{array}\right)\left[\operatorname{Pr}\left(E_{1}=1 \mid a_{1}\right)\right]^{k} .
$$

The conditional probability of a packet error sequence is written as

$$
\begin{aligned}
\operatorname{Pr} & \left(\beta_{1}=\ell_{1}, \beta_{2}=\ell_{2}, \ldots, \beta_{n}=\ell_{n} \mid a_{1}, a_{1+b}, \ldots, a_{1+(n-1) b}\right) \\
= & \sum_{k_{1}=\ell_{1}}^{b} \cdots \sum_{k_{n}=\ell_{n}}^{b} \prod_{i=1}^{n}(-1)^{k_{i}+\ell_{i}}\left(\begin{array}{c}
b \\
k_{i}
\end{array}\right) \\
& \times\left[\operatorname{Pr}\left(E_{1+(i-1) b}=1 \mid a_{1+(i-1) b}\right)\right]^{k_{i}} .
\end{aligned}
$$

For BFSK modulation with noncoherent demodulation

$\left[\operatorname{Pr}\left(e_{1+(i-1) b}=1 \mid a_{1+(i-1) b}\right)\right]^{k_{i}}=\left(\frac{1}{2}\right)^{k_{i}} e^{-\frac{E_{s}}{0 N_{0}} \frac{k_{i}}{2} a_{1+(i-1) b}^{2}}$.
We then write the probability of the packet error sequence for the DC model as

$$
\begin{aligned}
& \operatorname{Pr}\left(\beta_{1}=\ell_{1}, \beta_{2}=\ell_{2}, \cdots, \beta_{n}=\ell_{n}\right) \\
& =\sum_{k_{1}=\ell_{1}}^{b} \cdots \sum_{k_{n}=\ell_{n}}^{b} \prod_{i=1}^{n}(-1)^{k_{i}+\ell_{i}}\left(\begin{array}{c}
b \\
k_{i}
\end{array}\right)\left(\frac{1}{2}\right)^{k_{i}} \\
& \times \mathbf{E}\left[e^{-\frac{E_{s}}{N_{0}} \frac{k_{i}}{2} \sum_{i=1}^{n} a_{1+(i-1) b}^{2}}\right] \\
& =\sum_{k_{1}=\ell_{1}}^{b} \cdots \sum_{k_{n}=\ell_{n}}^{b} \prod_{i=1}^{n}(-1)^{k_{i}+\ell_{i}}\left(\begin{array}{c}
b \\
k_{i}
\end{array}\right)\left(\frac{1}{2}\right)^{k_{i}} \\
& \times \mathbf{E}\left[e^{-\frac{E_{s}}{N_{0}} \mathbf{G}^{\dagger} \mathbf{F G}}\right] \\
& =\sum_{k_{1}=\ell_{1}}^{b} \cdots \sum_{k_{n}=\ell_{n}}^{b} \prod_{i=1}^{n}(-1)^{k_{i}+\ell_{i}}\left(\begin{array}{c}
b \\
k_{i}
\end{array}\right)\left(\frac{1}{2}\right)^{k_{i}} \\
& \times \frac{\exp \left\{-\frac{E_{s}}{N_{0}} K_{R} \mathbf{1}^{T} \mathbf{F}\left(\left(K_{R}+1\right) \mathbf{I}+\frac{E_{s}}{N_{0}} \overline{\mathbf{C}} \mathbf{F}\right)^{-1} \mathbf{1}\right\}}{\operatorname{det}\left(\mathbf{I}+\frac{E_{s}}{N_{0}}\left(1+K_{R}\right)^{-1} \overline{\mathbf{C}} \mathbf{F}\right)} .
\end{aligned}
$$

\section{APPENDIX II}

\section{DERIVATION OF (21)}

Let

$$
P(s, z)=\frac{1-\varepsilon(1-p) s z-\varepsilon p z}{\left.1-(p+\varepsilon(1-p)) s z-(\varepsilon p+(1-p)) z+\varepsilon s z^{2}\right]} .
$$

Using the partial fraction technique described in [29, App.] to extract the coefficient of $P(s, z)$, we express $P(m, n)$ as

$$
P(m, n)=g(m, n)-\varepsilon(1-p) g(m-1, n-1)-\varepsilon p g(m, n-1)
$$

where

$$
\begin{aligned}
g(m, n)= & \frac{[p+\varepsilon(1-p)]^{m}[(1-p)+\varepsilon p]^{n-m}}{2^{n}} \\
& \times \sum_{j=0}^{\lfloor n / 2\rfloor} \sum_{\ell=0}^{\min \{m, j, n-m\}}\left(\begin{array}{c}
n+1 \\
2 j+1
\end{array}\right)\left(\begin{array}{l}
j \\
\ell
\end{array}\right)\left(\begin{array}{c}
n-2 \ell \\
m-\ell
\end{array}\right) \\
& \times\left[\frac{-4 \varepsilon}{[p+\varepsilon(1-p)][(1-p)+\varepsilon p]}\right]^{\ell}
\end{aligned}
$$

Defining $x=p+\varepsilon(1-p), \quad y=(1-p)+\varepsilon p, \quad$ and $m_{0}=$ $\min \{m, n-m\}$ and exchanging the order of summation in (26), we get

$$
g(m, n)=\frac{x^{m} y^{n-m}}{2^{n}} \sum_{\ell=0}^{m_{0}}\left(\begin{array}{c}
n-2 \ell \\
m-\ell
\end{array}\right)\left(\frac{-\varepsilon}{x y}\right)^{\ell} 4^{\ell} \sum_{j=\ell}^{\lfloor n / 2\rfloor}\left(\begin{array}{c}
n+1 \\
2 j+1
\end{array}\right)\left(\begin{array}{l}
j \\
\ell
\end{array}\right) .
$$


Since

$$
\sum_{j=\ell}^{\lfloor n / 2\rfloor}\left(\begin{array}{c}
n+1 \\
2 j+1
\end{array}\right)\left(\begin{array}{l}
j \\
\ell
\end{array}\right)=2^{n} 4^{-\ell}\left(\begin{array}{c}
n-\ell \\
\ell
\end{array}\right)
$$

the expression for $g(m, n)$ now becomes

$$
g(m, n)=x^{m} y^{n-m} \sum_{\ell=0}^{m_{0}}\left(\begin{array}{c}
n-2 \ell \\
m-\ell
\end{array}\right)\left(\begin{array}{c}
n-\ell \\
\ell
\end{array}\right)\left(\frac{-\varepsilon}{x y}\right)^{\ell} .
$$

Let

$$
\begin{aligned}
F(m, n) & =\sum_{\ell=0}^{m_{0}}\left(\begin{array}{c}
n-2 \ell \\
m-\ell
\end{array}\right)\left(\begin{array}{c}
n-\ell \\
\ell
\end{array}\right)\left(\frac{-\varepsilon}{x y}\right)^{\ell} \\
& =\sum_{\ell=0}^{m_{0}}\left[\prod_{j=\ell}^{m_{0}-1+\ell}(n-j)\right] \frac{(-\varepsilon / x y)^{\ell}}{\left(m_{0}-\ell\right) ! \ell !}
\end{aligned}
$$

for $n \geq 0$ and $m \geq 0$, and $F(m, n)=0$ for $n<0$ or $m<0$. Substituting (27) and (28) into (26), we express $P(m, n)$ as

$$
\begin{array}{r}
P(m, n)=x^{m} y^{n-m}\left[F(m, n)-\frac{\varepsilon(1-p)}{x}\right. \\
F(m-1, n-1) \\
\left.-\frac{\varepsilon p}{y} F(m, n-1)\right] .
\end{array}
$$

If $n \geq 2 m+1, F(m, n)$ and $F(m, n-1)$ are a summation of $m+1$ terms, and $F(m-1, n-1)$ has $m$ terms. These three quantities have several terms in common, and $P(m, n)$ becomes

$$
\begin{aligned}
P(m, n)= & x^{m} y^{n-m}\left\{\sum_{\ell=0}^{m} \frac{\left[\prod_{j=\ell+1}^{M-1+\ell}(n-j)\right]}{(m-\ell) ! \ell !}\left(\frac{-\varepsilon}{x y}\right)^{\ell}\right. \\
& \left.\times\left[\frac{(1-p)(n-\ell)}{y}-\frac{\varepsilon(1-p)(m-\ell)}{x}+\frac{\varepsilon p m}{y}\right]\right\} .
\end{aligned}
$$

\section{ACKNOWLEDGMENT}

The authors would like to thank the reviewers for providing constructive comments and suggestions that have improved the quality of this paper.

\section{REFERENCES}

[1] M. Zorzi, R. Rao, and L. B. Milstein, "ARQ error control for fading mobile radio channels," IEEE Trans. Veh. Technol., vol. 46, no. 2, pp. 445455, May 1997.

[2] F. Babich and G. Lombardi, "A Markov model for the mobile propagation channel," IEEE Trans. Veh. Technol., vol. 49, no. 1, pp. 63-73, Jan. 2000.

[3] M. R. Hueda and C. E. Rodríguez, "On the relationship between the block error and channel-state Markov models in transmissions over slowfading channels," IEEE Trans. Commun., vol. 52, no. 8, pp. 1269-1275, Aug. 2004.
[4] R. Annavajjala, A. Chockalingam, P. C. Cosman, and L. B. Milstein, "First-order Markov models for packet transmission on Rayleigh fading channels with DPDK/NCFSK modulation," in Proc. IEEE Int. Symp. Inf. Theory, Seattle, WA, Jul. 2006, pp. 2864-2868.

[5] Y. Yu and S. Miller, "A four-state Markov frame error model for the wireless physical layer," in Proc. IEEE Wireless Commun. Netw. Conf., Hong Kong, Mar. 2007, pp. 2055-2059.

[6] V. Tralli and M. Zorzi, "Markov models for the physical layer block error process in a WCDMA cellular system," IEEE Trans. Veh. Technol., vol. 54, no. 6, pp. 2102-2113, Nov. 2005.

[7] C. H. C. Leung, Y. Kikumoto, and S. A. Sorensen, "The throughput efficiency of the go-back-N ARQ scheme under Markov and related error structures," IEEE Trans. Commun., vol. 36, no. 2, pp. 231-234, Feb. 1988.

[8] P. M. Soni and A. Chockalingam, "Analysis of link-layer backoff schemes on point-to-point Markov fading links," IEEE Trans. Commun., vol. 51, no. 1, pp. 29-32, Jan. 2003.

[9] J. Yee and E. Weldon, "Evaluation of the performance of error-correcting codes on a Gilbert channel," IEEE Trans. Commun., vol. 43, no. 8, pp. 2316-2323, Aug. 1995.

[10] C. Pimentel, T. H. Falk, and L. Lisbôa, "Finite-state Markov modeling of correlated Rician-fading channels," IEEE Trans. Veh. Technol., vol. 53, no. 5, pp. 1491-1501, Sep. 2004.

[11] L. Zhong, F. Alajaji, and G. Takahara, "A model for correlated Rician fading channels based on a finite queue," IEEE Trans. Veh. Technol., vol. 57, no. 1, pp. 79-89, Jan. 2008.

[12] L. Wilhelmsson and L. B. Milstein, "On the effect of imperfect interleaving for the Gilbert-Elliott channel," IEEE Trans. Commun., vol. 47, no. 5, pp. 681-688, May 1999.

[13] F. Babich, O. Kelly, and G. Lombardi, "Generalized Markov modeling for flat fading," IEEE Trans. Commun., vol. 48, no. 4, pp. 547-551, Apr. 2000.

[14] W. Turin and R. van Nobelen, "Hidden Markov modeling of flat fading channels," IEEE J. Sel. Areas Commun., vol. 16, no. 9, pp. 1809-1817, Dec. 1998.

[15] W. Zhu and J. Garcia-Frias, "Stochastic context-free grammars and hidden Markov models for modeling of bursty channels," IEEE Trans. Veh. Technol., vol. 53, no. 3, pp. 666-676, May 2004.

[16] P. Sadeghi and P. Rapajic, "Capacity analysis for finite-state Markov mapping of flat-fading channels," IEEE Trans. Commun., vol. 53, no. 5, pp. 833-840, May 2005.

[17] C. Nicola, F. Alajaji, and T. Linder, "Decoding LDPC codes over binary channels with additive Markov noise," in Proc. 9th Can. Workshop Inf. Theory, Montreal, QC, Canada, Jun. 2005, pp. 187-190.

[18] J. Garcia-Frias, "Decoding of low-density parity-check codes over finitestate binary Markov channels," IEEE Trans. Commun., vol. 52, no. 11, pp. 1840-1843, Nov. 2004.

[19] A. W. Eckford, F. R. Kschischang, and S. Pasupathy, "Analysis of lowdensity parity-check codes for the Gilbert-Elliott channels," IEEE Trans. Inf. Theory, vol. 51, no. 11, pp. 3872-3889, Nov. 2005.

[20] L. Zhong, F. Alajaji, and G. Takahara, "A binary communication channel with memory based on a finite queue," IEEE Trans. Inf. Theory, vol. 53, no. 8, pp. 2815-2840, Aug. 2007.

[21] W. J. Gross, F. R. Kschischang, R. Koetter, and P. G. Gulak, "Applications of algebraic soft-decision decoding of Reed-Solomon codes," IEEE Trans. Commun., vol. 54, no. 7, pp. 1224-1234, Jul. 2006

[22] H. J. Kim, Y. G. Kim, I. Song, and J. D. Kim, "Difference threshold test for M-FSK signaling with Reed-Solomon coding and diversity combining in Rayleigh fading channels," IEEE Trans. Veh. Technol., vol. 54, no. 3 , pp. 977-982, May 2005.

[23] J. D. Choi, D.-S. Yoo, and W. E. Stark, "Performance limits of M-FSK with Reed-Solomon coding and diversity combining," IEEE Trans. Commun., vol. 50, no. 11, pp. 1787-1797, Nov. 2002.

[24] J. Lai and N. Mandayam, "Performance of Reed-Solomon codes for hybrid-ARQ over Rayleigh fading channels under imperfect interleaving," IEEE Trans. Commun., vol. 48, no. 10, pp. 1650-1659, Oct. 2000

[25] H. Labiod, "Performance of Reed Solomon error-correcting codes on fading channels," in Proc. IEEE Int. Conf. Pers. Wireless Commun., Jaipur, India, Feb. 1999, pp. 259-263.

[26] C. Pimentel and I. F. Blake, "Concatenated coding performance for FSK modulation on time-correlated Rician fading channels," IEEE Trans. Commun., vol. 46, no. 12, pp. 1610-1618, Dec. 1998.

[27] K. Sakakibara and J. Yamakita, "Performance comparison of imperfect symbol- and bit-interleaving of block codes over $\mathrm{GF}\left(2^{m}\right)$ on a Markovian channel," IEEE Trans. Wireless Commun., vol. 3, no. 1, pp. 269-277, Jan. 2004. 
[28] H. Al-Lawati, F. Alajaji, and C. Pimentel, "When can interleaving be avoided for Reed-Solomon coded binary Markov channels?" in Proc. IEEE Pacific Rim Conf. Commun., Comput. Signal Process., Victoria, BC, Canada, Aug. 2007, pp. 530-533.

[29] C. Pimentel and I. F. Blake, "Enumeration of Markov chains and burst error statistics for finite state channel models," IEEE Trans. Veh. Technol., vol. 48, no. 2, pp. 415-428, Mar. 1999.

[30] A. I. Drukarev and K. P. Yiu, "Performance of error-correcting codes on channels with memory," IEEE Trans. Commun., vol. COM-34, no. 6, pp. 513-521, Jun. 1986.

[31] S. G. Srinivasa, P. Lee, and S. W. McLaughlin, "Post-error correcting code modeling of burst channels using hidden Markov models with applications to magnetic recording," IEEE Trans. Magn., vol. 43, no. 2, pp. 572-579, Feb. 2007.

[32] C. Pimentel and I. F. Blake, "Modeling burst channels using partitioned Fritchman's Markov models," IEEE Trans. Veh. Technol., vol. 47, no. 3, pp. 885-899, Aug. 1998.

[33] C. Tan and N. C. Beaulieu, "On first-order Markov modeling for the Rayleigh fading channel," IEEE Trans. Commun., vol. 48, no. 12, pp. 2032-2040, Dec. 2000.

[34] W. Kumwilaisak, C. Kuo, and D. Wu, "Fading channel modeling via variable-length Markov chain technique," IEEE Trans. Veh. Technol., vol. 57, no. 3, pp. 1338-1358, May 2008.

[35] A. E. Drougas, A. D. Panagopoulos, and P. G. Cottis, "Stochastic verification of the first-order Markovian assumption of rain attenuation for satellite channel dynamic modeling," IEEE Commun. Lett., vol. 12, no. 9, pp. 663-665, Sep. 2008.

[36] J. K. Wolf, "ECC performance of interleaved RS codes with burst errors," IEEE Trans. Magn., vol. 34, no. 1, pp. 75-79, Jan. 1998.

[37] T. Li and O. M. Collins, "A successive decoding strategy for channels with memory," IEEE Trans. Inf. Theory, vol. 53, no. 2, pp. 628-646, Feb. 2007.

[38] H. Al-Lawati and F. Alajaji, "On decoding binary perfect and quasiperfect codes over Markov noise channels," IEEE Trans. Commun., vol. 57, Apr. 2009

[39] W. Griffiths, H.-J. Zepernick, and M. Caldera, "APP decoding of non-binary block codes on Gilbert-Elliott channels using generalized weight polynomials," in Proc. Int. Symp. Inf. Theory Appl., Auckland, New Zealand, Dec. 2008, pp. 1077-1082.

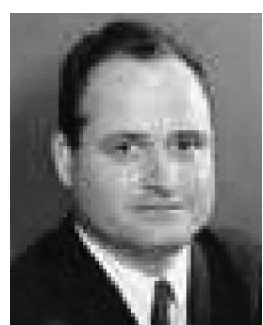

Cecilio Pimentel was born in Recife, Brazil, in 1966. He received the B.Sc. degree from the Federal University of Pernambuco, Recife, in 1987, the M.Sc. degree from the Catholic University of Rio de Janeiro, Rio de Janeiro, Brazil, in 1990, and the Ph.D. degree from the University of Waterloo, Waterloo, ON, Canada, in 1996, all in electrical engineering.

Since October 1996, he has been with the Department of Electronics and Systems, Federal University of Pernambuco, where he is currently an Associate Professor. From 2007 to 2008, he was a Visiting Research Scholar with the Department of Mathematics and Statistics, Queen's University, Kingston, ON, Canada. His research interests include digital communications, information theory, and error-correcting coding.

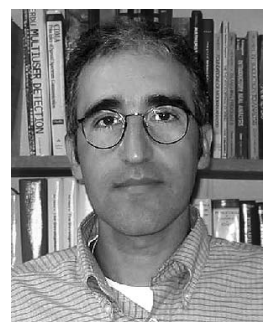

Fady Alajaji (S'90-M'94-SM'00) was born in Beirut, Lebanon, on May 1, 1966. He received the B.E. degree (with distinction) from the American University of Beirut and the M.Sc. and Ph.D. degrees from the University of Maryland, College Park, all in electrical engineering, in 1988, 1990, and 1994, respectively.

In 1994, he held a postdoctoral appointment with the Institute for Systems Research, University of Maryland. In 1995, he joined the Department of Mathematics and Statistics, Queen's University, Kingston, ON, Canada, where he is currently a Professor of mathematics and engineering. Since 1997, he has held a cross-appointment position with the Department of Electrical and Computer Engineering at the same university. His research interests include information theory, joint source-channel coding, error control coding, data compression, and digital communications.

Dr. Alajaji currently serves as Area Editor and Editor for Source and SourceChannel Coding for the IEEE TRANSACTIONS ON COMMUNICATIONS. He served as Cochair of the 1999 Canadian Workshop on Information Theory, Cochair of the Technical Program Committee (TPC) of the 2004 Biennial Symposium on Communications, and TPC member of several international conferences and workshops. He was the recipient of the Premier's Research Excellence Award from the Province of Ontario. 